\title{
Caffeine Prevalence, Daily Consumption, and Factors Associated with Use among Active Duty United States Military Personnel
}

Joseph J Knapik ( $\nabla$ joseph.j.knapik.civ@mail.mil )

US Army Research Institute of Environmental Medicine https://orcid.org/0000-0002-1568-1860

Ryan A Steelman

US Army Public Health Center

Daniel W. Trone

Naval Health Research Center

Emily K. Farina

US Army Research Institute of Environmental Medicine

Harris R. Lieberman

US Army Research Institute of Environmental Medicine

\section{Research}

Keywords: coffee, tea, soda, energy drinks

Posted Date: December 14th, 2021

DOI: https://doi.org/10.21203/rs.3.rs-1138093/v1

License: @ (1) This work is licensed under a Creative Commons Attribution 4.0 International License. Read Full License 


\section{Abstract}

Background: Although representative data on caffeine intake in Americans are available these data do not include US service members (SMs). The few previous investigations in military personnel largely involve convenience samples. This cross-sectional study examined prevalence of caffeine use, daily consumption, and factors associated with use among United States active duty military service members (SMs).

Methods: A stratified random sample of 200,000 SMs were asked to complete a questionnaire on their personal characteristics and consumption of caffeinated products. Eighteen percent $(n=26,680)$ of successfully contacted SMs $(n=146,365)$ completed the questionnaire.

Results: Overall, $87 \%$ reported consuming caffeinated products $\geq 1$ time/week. Mean \pm standard error per-capita consumption (all participants) was $218 \pm 2$ and $167 \pm 3 \mathrm{mg} /$ day for men and women, respectively. Caffeine consumers ingested $243 \pm 2 \mathrm{mg} /$ day ( $251 \pm 2$ $\mathrm{mg} /$ day men, $195 \pm 3 \mathrm{mg} /$ day women). On a body-weight basis, men and women consumed respectively similar caffeine amounts ( 2.93 vs $2.85 \mathrm{mg} /$ day $/ \mathrm{kg} ; \mathrm{p}=0.12$ ). Among individual caffeinated products, coffee had the highest prevalence $(68 \%)$, followed by sodas (42\%), teas (29\%), energy drinks (29\%) and gums/candy/medications (4\%). In multivariable logistic regression, characteristics independently associated with higher use prevalence ( $\geq 1$ time/week) included female gender, older age, white race/ethnicity, higher body mass index, tobacco use or former use, greater alcohol intake, and higher enlisted or officer rank.

Conclusion: Compared to National Health and Nutrition Survey Examination data, daily consumption (mg/day) by SMs was higher, perhaps reflecting higher mental and physical occupational demands on SMs.

\section{Background}

Caffeine is a widely consumed psychoactive stimulant ingested in various beverages including coffees, teas, sodas, and energy drinks. About $90 \%$ of United States (US) adults consume caffeinated products with little difference between men and women in how frequently the products are ingested [1, 2]. Among caffeine consumers, the average caffeine intake is about $211 \mathrm{mg} /$ day [1]. Although there are cases where consumption of very high dosages of caffeine has led to seizures, transient cardiovascular problems, and even deaths [3, 4], comprehensive reviews have concluded that consumption of $<400 \mathrm{mg} /$ day is generally safe, enhances certain aspects of mental, physical, and occupational performance, and may confer other health benefits [5-7]. Among healthy adults, moderate coffee consumption has been reported to be associated with reduced risk of certain health conditions including chronic liver disease, gout, Parkinson's disease, Alzheimer's disease, Type 2 diabetes, certain types of cancers, all-cause mortality, and cause-specific mortality [5, 8 , 9]. However, concerns about caffeine use by pregnant women and increased consumption of energy drinks by young adults has been expressed [5, 10]. For pregnant women, caffeine dosages in the range of $<200$ to $300 \mathrm{mg} / \mathrm{day}$ are recommended [7] because of increased risk for pregnancy-related adverse effects (low birth weight, pregnancy loss, childhood leukemia) [8]. Data from the nationally-representative National Health and Nutrition Examination Survey (NHANES) indicate caffeine consumption from energy drinks increased from 2001 to 2016 in 19- to 39-year-olds, but was relatively low [10, 11]. Furthermore, total caffeine consumption did not significantly increase, as there was a concurrent reduction in caffeine intake from sodas in 2001-2010 [11].

Although representative data on caffeine intake in Americans are available [1, 2, 11-15], these data do not include US service members (SMs). Among civilians, caffeine use varies depending on occupation; working in "legal" and "management" occupations was associated with greater caffeine intake than the average of all other occupations investigated [15]. Military personnel must engage in a number of physically- and cognitively-demanding tasks such as intelligence gathering, tactical planning, guard duty, lengthy marches with heavy backpacks, lifting and carrying substantial loads, movement over and under obstacles, and other operational tasks that can require lengthy and intense activity. Furthermore, military work hours are not regulated (restricted) by the labor laws governing the civilian population in the US, and active duty SMs are employed full-time. The extensive physical demands placed on SMs include early morning physical training, and limited sleep during training, operations, and deployments. Studies of US SMs have found that approximately $70 \%$ sleep $<6$ hours per night and only $~ 30 \%$ are getting the recommended $7-8$ hours of sleep per night [16]. This contrasts with studies of the US civilian population where $72 \%$ of civilians sleep $\geq 7$ hours per night and only $28 \%$ sleep $<6$ hours/night $[16,17]$. Demanding tasks and lack of adequate sleep may lead SMs to consume more caffeinated substances than the general population. In particularly demanding circumstances, such as combat environments, and in populations such as aviators, caffeine use is particularly high $[18,19]$. 
Caffeine consumption in Army [20], Navy/Marine Corps [21] and Air Force [22] personnel has been investigated in separate surveys by our group, usually in convenience samples, and was higher than the civilian population [1, 2, 15, 20-22]. The purpose of the current investigation was to examine the more recent prevalence of caffeine use, amount of caffeine consumption, and factors associated with use in a single, large, stratified random sample of US military personnel SMs from all services.

\section{Materials And Methods}

This investigation involved a cross-sectional survey completed by US military SMs and was part of a larger study involving the health effects of dietary supplements [23]. The Naval Health Research Center's institutional review board approved the study protocol, and SMs consented to participate by signing an informed consent document. Investigators adhered to policies and procedures for protection of human subjects as prescribed by Department of Defense Instruction 3216.01, and the research was conducted in adherence with provisions of Title 32, Code of Federal Regulations, Part 219.

\section{Sampling Frame and Solicitation Procedures}

Details of the sampling frame, solicitation of SMs, participant flow through the study, and response bias were described previously [23]. Briefly, investigators requested a random sample of $200,000 \mathrm{SMs}$, stratified by sex ( $88 \%$ male and $12 \%$ female) and branch of service (Army 36\%, Air Force 24\%, Marine Corps 15\%, and Navy 25\%), from the Defense Manpower Data Center. Recruitment of SMs into the study from this random sample involved a maximum of 12 sequential contacts. Investigators sent the prospective participant an introductory postal letter with a $\$ 1$ pre-incentive designed to increase the response rate $[24,25]$. The letter described the survey, included a link to a secure website, and a unique login that could be used to access the web-based survey and electronically sign the consent form. SMs who did not initially complete the survey were sent a follow-up email message after 10 days, and a postcard after 3 weeks as a reminder. If the SM did not respond after having received the postcard, he/she received up to seven additional email reminders and three postcards evenly distributed during the time the survey was open. Reminders were sent only to those who had not responded. All postal and on-line contacts stated the SM could decline participation at any time and be removed from the contact list. Recruitment began in December 2018; after August 2019, no further recruitment was conducted, and no surveys were accepted.

\section{Survey Description}

The questionnaire was administered online and was designed to characterize participants and quantify the frequency and amount of caffeinated products they consumed in the last 6 months. To characterize participants, there were questions on demographics (gender, age, education, race/ethnicity, height, weight), lifestyle factors (exercise, tobacco use, alcohol consumption, sleep), and military characteristics (rank, occupation assignment, service branch). Participants were asked to select serving sizes for coffee, tea, and soft drinks and how often they were consumed. Sizes available for selection included 8, 12, 16, 20, and 24+ ounces; frequencies available for selection included per day, week, month, or year; and number of times consumed available for selection ranged from 0 to 8 for each frequency option. For energy shots and energy beverages, participants provided the number of bottles or packets $(0$ to 16$)$ and the frequency (per day, week, month, or year). For caffeinated candy and gum, participants provided the number of candies or sticks of gum (0 to 16) and the frequency (per day, week, month, or year). For caffeinated medications, participants provided the number of pills consumed ( 0 to 16$)$ and the frequency (per day, week, month, or year). Examples were provided for energy shots (e.g. 5Hour Energy), energy beverages (e.g., Red Bull, Monster, Full Throttle), caffeinated candy/gum (e.g., Military Energy, GoFastGum), and caffeinated medications (e.g., Bayer, Excedrin, No Doz).

\section{Data Analysis}

Statistical analyses were conducted using the Statistical Package for the Social Sciences (Version 21.0.0.0, 2019, IBM Corporation). Caffeine products were grouped into five types: 1) coffee; 2) tea; 3 ) sodas; 4) energy drinks (energy shots and energy beverages combined); 5) caffeinated gums/medications (candies, gum, and medications combined). All types were then combined to determine an aggregated caffeine intake (i.e., any caffeine product).

An individual who used any caffeinated product $\geq 1$ time/week was considered a caffeine consumer. Caffeine consumption (mg/day) was calculated based on the serving size and the frequency of consumption using publicly available databases and estimates of caffeine content in various products [26,27]. One ounce of coffee, tea, and soda was considered to contain 12, 6, and $3 \mathrm{mg}$ caffeine per ounce, respectively. Energy drinks and energy shots were considered to contain 160 and $200 \mathrm{mg}$ caffeine, respectively. Energy gum/candy and medication were considered to contain 50 and $100 \mathrm{mg}$ caffeine, respectively.

Page $3 / 25$ 
Body mass index (BMI) was calculated as self-reported weight/height ${ }^{2}\left(\mathrm{~kg} / \mathrm{m}^{2}\right)$. Weekly duration of aerobic training or resistance training (minutes/week) was calculated by multiplying weekly exercise frequency (sessions/week) by the duration of training (minutes/session). Tobacco users were defined as individuals who reported using any tobacco products in the past week; former tobacco users were defined as those who reported having used tobacco products in the past but had quit within the last year or earlier.

Prevalence of consumption $\geq 1$ time/week (\%) with standard errors (SE) was calculated for each caffeine product type individually and for all caffeine products in aggregate (i.e., any caffeine). Chi-square statistics were used to examine prevalence differences across various strata of demographic, lifestyle, and military characteristics. A one-way analysis of variance (ANOVA) examined differences in daily caffeine consumption (mg/day) across strata of these characteristics. For ordinal variables (i.e., age, education, BMI, aerobic training duration, resistance training duration, alcohol intake, sleep), tests for linear trend, Mantel-Haenszel statistics, and ANOVA linear contrasts were also performed. Since some participants did not complete all of the questions, tables present the number of participants for each variable. Multivariable logistic regression was used to examine associations between use and non-use of caffeine products ( $\geq 1$ time/week) and independent variables that included the demographic, lifestyle, and military characteristics. Six separate regression models were developed for each caffeine product type: "any caffeine," coffee, tea, soda, energy drinks, and caffeinated gum/medications. A one-way ANOVA for linear trend compared caffeine consumption across age groups in men and women separately. Self-reported sleep duration was not included in the multivariable analyses because only $78 \%$ of SMs responded to this question. Since multivariable analysis requires complete data on all variables, including sleep duration would have removed a large number of SMs from the multivariable analyses.

\section{Results}

From the initial list of 200,000 potential volunteers, 146,365 (73\%) were successfully contacted (i.e., no returned postal mail). Of these, $26,680(18.2 \%)$ signed the informed consent and completed the questionnaire online.

\section{Caffeine Use Prevalence}

Table 1 provides prevalence of reported caffeine consumption by demographic, lifestyle, and military characteristics. Overall, $87 \%$ of participants reported using products containing caffeine $\geq 1$ time per week, with coffee and soda being the most frequently consumed. For energy drinks and energy shots considered individually, use (prevalence \pm SE) was $28.4 \pm 0.3 \%$ and $2.4 \pm 0.1 \%$, respectively; for gums/candies and medications individually, use was $0.9 \pm 0.1 \%$ and $3.5 \pm 0.1 \%$, respectively. 
Table 1

Prevalence $(\% \pm$ SE) of reported caffeine consumption ( $\geq 1$ time/week) in military personnel by demographic, lifestyle, and military characteristics

\begin{tabular}{|c|c|c|c|c|c|c|c|}
\hline Variable & Strata & $\begin{array}{l}\text { Any } \\
\text { Caffeine }\end{array}$ & Coffee & Tea & Soda & $\begin{array}{l}\text { Energy } \\
\text { Drink }\end{array}$ & $\begin{array}{l}\text { Gums \& } \\
\text { Medications }\end{array}$ \\
\hline Cohort & All $(n=26,680)$ & $86.9 \pm 0.2$ & $68.1 \pm 0.3$ & $29.2 \pm 0.3$ & $41.9 \pm 0.3$ & $29.3 \pm 0.3$ & $4.3 \pm 0.1$ \\
\hline \multirow[t]{3}{*}{ Gender } & Men $(n=23,038)$ & $87.0 \pm 0.2$ & $68.3 \pm 0.3$ & $27.0 \pm 0.3$ & $43.1 \pm 0.3$ & $31.3 \pm 0.3$ & $3.9 \pm 0.1$ \\
\hline & Women $(n=3,642)$ & $86.1 \pm 0.6$ & $66.9 \pm 0.8$ & $43.5 \pm 0.8$ & $33.8 \pm 0.8$ & $16.8 \pm 0.6$ & $6.5 \pm 0.4$ \\
\hline & p-value (chi-square) & 0.13 & 0.10 & $<0.01$ & $<0.01$ & $<0.01$ & $<0.01$ \\
\hline \multirow[t]{6}{*}{ Age } & $18-24$ years & $77.6 \pm 0.6$ & $52.7 \pm 0.7$ & $24.9 \pm 0.6$ & $40.8 \pm 0.7$ & $34.7 \pm 0.7$ & $3.5 \pm 0.3$ \\
\hline & $(I I-4,000)$ & $85.1 \pm 0.5$ & $66.1 \pm 0.6$ & $28.0 \pm 0.6$ & $38.6 \pm 0.7$ & $34.2 \pm 0.6$ & $3.6 \pm 0.2$ \\
\hline & $(\mathrm{n}=5,580)$ & $89.5 \pm 0.3$ & $72.2 \pm 0.5$ & $29.3 \pm 0.4$ & $42.7 \pm 0.5$ & $30.7 \pm 0.4$ & $4.7 \pm 0.2$ \\
\hline & $\begin{array}{l}30-39 \text { years } \\
(n=11,030)\end{array}$ & $91.4 \pm 0.4$ & $75.1 \pm 0.6$ & $34.0 \pm 0.7$ & $44.8 \pm 0.7$ & $17.3 \pm 0.5$ & $4.8 \pm 0.3$ \\
\hline & $\begin{array}{l}\geq 40 \text { years } \\
(n=5,275)\end{array}$ & & & & & & \\
\hline & $\begin{array}{l}\text { p-value (chi } \\
\text { square/trend) }\end{array}$ & $<0.01 /<0.01$ & $<0.01 /<0.01$ & $<0.01 /<0.01$ & $<0.01 /<0.01$ & $<0.01 /<0.01$ & $<0.01 /<0.01$ \\
\hline \multirow[t]{5}{*}{ Education } & \multirow{2}{*}{$\begin{array}{l}\text { Some high } \\
\text { school/High school } \\
\text { graduate }(n=3,879)\end{array}$} & $79.9 \pm 0.6$ & $55.0 \pm 0.8$ & $22.9 \pm 0.7$ & $45.0 \pm 0.8$ & $38.8 \pm 0.8$ & $4.1 \pm 0.3$ \\
\hline & & $86.4 \pm 0.3$ & $67.1 \pm 0.4$ & $28.2 \pm 0.4$ & $43.1 \pm 0.5$ & $35.1 \pm 0.5$ & $4.8 \pm 0.2$ \\
\hline & $\begin{array}{l}\text { Some } \\
\text { college/Associate's } \\
\text { degree }(n=11,378)\end{array}$ & $89.6 \pm 0.3$ & $73.5 \pm 0.4$ & $32.3 \pm 0.4$ & $39.6 \pm 0.5$ & $20.3 \pm 0.4$ & $3.8 \pm 0.2$ \\
\hline & $\begin{array}{l}\text { Bachelor's/Graduate } \\
\text { degree }(n=11,417)\end{array}$ & & & & & & \\
\hline & $\begin{array}{l}\text { p-value (chi } \\
\text { square/trend) }\end{array}$ & $<0.01 /<0.01$ & $<0.01 /<0.01$ & $<0.01 /<0.01$ & $<0.01 /<0.01$ & $<0.01 /<0.01$ & $<0.01 / 0.06$ \\
\hline \multirow{5}{*}{$\begin{array}{l}\text { Race / } \\
\text { Ethnicity }\end{array}$} & White $(n=16,316)$ & $90.9 \pm 0.2$ & $73.0 \pm 0.3$ & $29.0 \pm 0.4$ & $44.4 \pm 0.4$ & $31.4 \pm 0.4$ & $4.5 \pm 0.2$ \\
\hline & Hispanic $(n=4,227)$ & $83.5 \pm 0.6$ & $65.2 \pm 0.7$ & $25.2 \pm 0.8$ & $39.2 \pm 0.8$ & $30.4 \pm 0.7$ & $3.6 \pm 0.3$ \\
\hline & Black $(n=2,966)$ & $72.2 \pm 0.8$ & $46.5 \pm 0.9$ & $30.7 \pm 0.8$ & $34.4 \pm 0.9$ & $18.8 \pm 0.7$ & $4.6 \pm 0.4$ \\
\hline & Other $(n=3,171)$ & $84.5 \pm 0.6$ & $67.1 \pm 0.8$ & $34.6 \pm 0.8$ & $39.4 \pm 0.9$ & $27.1 \pm 0.8$ & $3.7 \pm 0.3$ \\
\hline & p-value (chi-square) & $<0.01$ & $<0.01$ & $<0.01$ & $<0.01$ & $<0.01$ & 0.03 \\
\hline \multirow{5}{*}{$\begin{array}{l}\text { Body mass } \\
\text { index }\end{array}$} & \multirow{2}{*}{$\begin{array}{l}<25.0 \mathrm{~kg} / \mathrm{m}^{2} \\
(\mathrm{n}=7,856)\end{array}$} & $84.4 \pm 0.4$ & $64.9 \pm 0.5$ & $30.6 \pm 0.5$ & $39.4 \pm 0.6$ & $24.7 \pm 0.5$ & $3.5 \pm 0.2$ \\
\hline & & $87.5 \pm 0.3$ & $69.4 \pm 0.4$ & $28.3 \pm 0.4$ & $41.2 \pm 0.4$ & $30.3 \pm 0.4$ & $4.2 \pm 0.2$ \\
\hline & $\begin{array}{l}25.0-29.9 \mathrm{~kg} / \mathrm{m}^{2} \\
(\mathrm{n}=13,897)\end{array}$ & $89.4 \pm 0.5$ & $69.6 \pm 0.7$ & $29.3 \pm 0.7$ & $48.4 \pm 0.8$ & $34.3 \pm 0.7$ & $5.9 \pm 0.3$ \\
\hline & $\underset{(n=4,424)}{\geq 30.0 \mathrm{~kg} / \mathrm{m}^{2}}$ & & & & & & \\
\hline & $\begin{array}{l}\text { p-value (chi } \\
\text { square/trend) }\end{array}$ & $<0.01 /<0.01$ & $<0.01 /<0.01$ & $<0.01 / 0.30$ & $<0.01 /<0.01$ & $<0.01 /<0.01$ & $<0.01 /<0.01$ \\
\hline
\end{tabular}




\begin{tabular}{|c|c|c|c|c|c|c|c|}
\hline Variable & Strata & $\begin{array}{l}\text { Any } \\
\text { Caffeine }\end{array}$ & Coffee & Tea & Soda & $\begin{array}{l}\text { Energy } \\
\text { Drink }\end{array}$ & $\begin{array}{l}\text { Gums \& } \\
\text { Medications }\end{array}$ \\
\hline \multirow{6}{*}{$\begin{array}{l}\text { Aerobic } \\
\text { exercise } \\
\text { duration }\end{array}$} & $\leq 90 \mathrm{~min} / \mathrm{wk}$ & $84.0 \pm 0.4$ & $66.5 \pm 0.6$ & $27.1 \pm 0.5$ & $42.5 \pm 0.6$ & $28.3 \pm 0.5$ & $4.3 \pm 0.2$ \\
\hline & $(n=/, 286)$ & $90.0 \pm 0.4$ & $71.4 \pm 0.5$ & $31.1 \pm 0.5$ & $45.7 \pm 0.6$ & $28.9 \pm 0.5$ & $4.2 \pm 0.2$ \\
\hline & $\begin{array}{l}91-180 \mathrm{~min} / \mathrm{wk} \\
(\mathrm{n}=7,285)\end{array}$ & $88.9 \pm 0.4$ & $70.5 \pm 0.6$ & $30.1 \pm 0.6$ & $42.4 \pm 0.6$ & $30.1 \pm 0.6$ & $4.0 \pm 0.3$ \\
\hline & $\begin{array}{l}181-300 \mathrm{~min} / \mathrm{wk} \\
(\mathrm{n}=5,869)\end{array}$ & $84.9+0.5$ & $65.6 \pm 0.6$ & $28.7 \pm 0.6$ & $36.2 \pm 0.6$ & $30.4 \pm 0.6$ & $4.5 \pm 0.3$ \\
\hline & $\underset{(\mathrm{n}=6,240)}{\geq 301 \mathrm{~min} / \mathrm{wk}}$ & & & & & & \\
\hline & $\begin{array}{l}\text { p-value (chi } \\
\text { square/trend) }\end{array}$ & $<0.01 / 0.49$ & $<0.01 / 0.62$ & $<0.01 / 0.09$ & $<0.01 /<0.01$ & $0.03 /<0.01$ & $0.57 / 0.64$ \\
\hline \multirow{6}{*}{$\begin{array}{l}\text { Resistance } \\
\text { training } \\
\text { frequency }\end{array}$} & $\leq 45 \mathrm{~min} / \mathrm{wk}$ & $85.5 \pm 0.4$ & $66.4 \pm 0.5$ & $30.9 \pm 0.5$ & $47.4 \pm 0.6$ & $24.2 \pm 0.5$ & $4.5 \pm 0.2$ \\
\hline & $(\mathrm{n}=/, / / / 6)$ & $90.1 \pm 0.4$ & $71.7 \pm 0.6$ & $32.1 \pm 0.6$ & $46.0 \pm 0.6$ & $26.7 \pm 0.6$ & $4.3 \pm 0.3$ \\
\hline & $\begin{array}{l}46-135 \mathrm{~min} / \mathrm{wk} \\
(\mathrm{n}=6,257)\end{array}$ & $89.0 \pm 0.4$ & $71.3 \pm 0.6$ & $29.3 \pm 0.6$ & $39.5 \pm 0.6$ & $31.1 \pm 0.6$ & $4.2 \pm 0.3$ \\
\hline & $\begin{array}{l}136-300 \mathrm{~min} / \mathrm{wk} \\
(\mathrm{n}=6,581)\end{array}$ & $82.9 \pm 0.5$ & $63.1 \pm 0.6$ & $24.0 \pm 0.5$ & $33.0 \pm 0.6$ & $36.8 \pm 0.6$ & $4.0 \pm 0.3$ \\
\hline & $\underset{(n=6,066)}{\geq 301 \mathrm{~min} / \mathrm{wk}}$ & & & & & & \\
\hline & $\begin{array}{l}\text { p-value (chi } \\
\text { square/trend) }\end{array}$ & $<0.01 /<0.01$ & $<0.01 /<0.01$ & $<0.01 /<0.01$ & $<0.01 /<0.01$ & $<0.01 /<0.01$ & $0.46 / 0.11$ \\
\hline \multirow[t]{4}{*}{ Smoking } & $\operatorname{Never}(n=16,706)$ & $84.9 \pm 0.3$ & $63.7 \pm 0.4$ & $29.7 \pm 0.4$ & $39.5 \pm 0.4$ & $24.5 \pm 0.3$ & $4.0 \pm 0.2$ \\
\hline & Former $(n=4,767)$ & $93.9 \pm 0.3$ & $79.9 \pm 0.6$ & $29.4 \pm 0.7$ & $44.1 \pm 0.7$ & $35.0 \pm 0.7$ & $5.2 \pm 0.3$ \\
\hline & Smoker $(n=4,511)$ & $93.6 \pm 0.4$ & $77.1 \pm 0.6$ & $29.9 \pm 0.7$ & $51.7 \pm 0.7$ & $43.6 \pm 0.7$ & $4.8 \pm 0.3$ \\
\hline & p-value (chi-square) & $<0.01$ & $<0.01$ & 0.88 & $<0.01$ & $<0.01$ & $<0.01$ \\
\hline \multirow{4}{*}{$\begin{array}{l}\text { Smokeless } \\
\text { tobacco use }\end{array}$} & Never $(n=20,378)$ & $86.4 \pm 0.2$ & $66.1 \pm 0.3$ & $30.6 \pm 0.3$ & $41.6 \pm 0.3$ & $26.2 \pm 0.3$ & $4.3 \pm 0.1$ \\
\hline & Former $(n=2,047)$ & $95.7 \pm 0.4$ & $82.9 \pm 0.8$ & $27.1 \pm 1.0$ & $43.7 \pm 1.1$ & $38.1 \pm 1.1$ & $4.8 \pm 0.5$ \\
\hline & Smoker $(n=3,114)$ & $94.3 \pm 0.4$ & $79.2 \pm 0.7$ & $26.4 \pm 0.8$ & $48.5 \pm 0.9$ & $47.8 \pm 0.9$ & $4.6 \pm 0.4$ \\
\hline & $\mathrm{p}$-value (chi-square) & $<0.01$ & $<0.01$ & $<0.01$ & $<0.01$ & $<0.01$ & 0.43 \\
\hline \multirow{6}{*}{$\begin{array}{l}\text { Alcohol } \\
\text { intake }\end{array}$} & $0 \mathrm{ml} / \mathrm{wk}(\mathrm{n}=8,372)$ & $73.8 \pm 0.5$ & $50.8 \pm 0.5$ & $23.3 \pm 0.5$ & $35.1 \pm 0.5$ & $23.9 \pm 0.5$ & $3.8 \pm 0.2$ \\
\hline & $0.23-24.85 \mathrm{ml} / \mathrm{wk}$ & $89.5 \pm 0.4$ & $70.2 \pm 0.6$ & $31.4 \pm 0.6$ & $41.2 \pm 0.6$ & $25.3 \pm 0.6$ & $3.9 \pm 0.2$ \\
\hline & $(n=0,132)$ & $93.3 \pm 0.3$ & $76.0 \pm 0.5$ & $31.5 \pm 0.6$ & $45.1 \pm 0.6$ & $31.9 \pm 0.6$ & $4.4 \pm 0.3$ \\
\hline & $\begin{array}{l}24.86-11.69 \mathrm{ml} / \mathrm{wk} \\
(\mathrm{n}=6,108)\end{array}$ & $95.9 \pm 0.3$ & $81.9 \pm 0.5$ & $32.9 \pm 0.6$ & $48.7 \pm 0.6$ & $38.3 \pm 0.6$ & $5.0 \pm 0.3$ \\
\hline & $\underset{(\mathrm{n}=6,067)}{\geq 71.70 \mathrm{ml} / \mathrm{wk}}$ & & & & & & \\
\hline & $\begin{array}{l}\text { p-value (chi } \\
\text { square/trend) }\end{array}$ & $<0.01 /<0.01$ & $<0.01 /<0.01$ & $<0.01 /<0.01$ & $<0.01 /<0.01$ & $<0.01 /<0.01$ & $<0.01 /<0.01$ \\
\hline \multirow[t]{5}{*}{ Sleep } & $<4 \mathrm{hr} / \mathrm{night}$ & $84.2 \pm 1.1$ & $65.4 \pm 1.4$ & $31.6 \pm 1.4$ & $43.7 \pm 1.5$ & $35.3 \pm 1.4$ & $8.2 \pm 0.8$ \\
\hline & & $90.9 \pm 0.3$ & $71.5 \pm 0.5$ & $30.6 \pm 0.5$ & $46.7 \pm 0.5$ & $34.7 \pm 0.5$ & $5.1 \pm 0.2$ \\
\hline & $\begin{array}{l}5-6 \mathrm{hr} / \mathrm{nIght} \\
(\mathrm{n}=10,027)\end{array}$ & $87.9 \pm 0.3$ & $69.3 \pm 0.5$ & $29.0 \pm 0.5$ & $40.8 \pm 0.5$ & $26.2 \pm 0.4$ & $3.3 \pm 0.2$ \\
\hline & $\begin{array}{l}\geq 7 \mathrm{hr} / \mathrm{night} \\
(\mathrm{n}=9,702)\end{array}$ & & & & & & \\
\hline & $\begin{array}{l}\mathrm{p} \text {-value (chi- } \\
\text { square/linear trend) }\end{array}$ & $<0.01 / 0.01$ & $<0.01 / 0.48$ & $0.02 /<0.01$ & $<0.01 /<0.01$ & $<0.01 /<0.01$ & $<0.01 /<0.01$ \\
\hline
\end{tabular}




\begin{tabular}{|c|c|c|c|c|c|c|c|}
\hline Variable & Strata & $\begin{array}{l}\text { Any } \\
\text { Caffeine }\end{array}$ & Coffee & Tea & Soda & $\begin{array}{l}\text { Energy } \\
\text { Drink }\end{array}$ & $\begin{array}{l}\text { Gums \& } \\
\text { Medications }\end{array}$ \\
\hline \multirow[t]{9}{*}{ Rank } & \multirow{2}{*}{$\begin{array}{l}\text { Junior enlisted } \\
(n=2,496)\end{array}$} & $74.9 \pm 0.9$ & $49.8 \pm 1.0$ & $24.7 \pm 0.9$ & $40.5 \pm 1.0$ & $33.1 \pm 0.9$ & $3.4 \pm 0.4$ \\
\hline & & $85.2 \pm 0.3$ & $64.5 \pm 0.4$ & $27.8 \pm 0.4$ & $42.8 \pm 0.5$ & $36.4 \pm 0.4$ & $4.7 \pm 0.2$ \\
\hline & $\begin{array}{l}\text { Mid enlisted } \\
(\mathrm{n}=11,609)\end{array}$ & $91.0 \pm 0.4$ & $74.4 \pm 0.7$ & $28.9 \pm 0.7$ & $44.7 \pm 0.8$ & $29.6 \pm 0.7$ & $4.9 \pm 0.3$ \\
\hline & \multirow{2}{*}{$\begin{array}{l}\text { Senior enlisted } \\
(n=4,365)\end{array}$} & $93.2 \pm 1.0$ & $78.1 \pm 1.7$ & $29.7 \pm 1.9$ & $39.2 \pm 2.0$ & $27.3 \pm 1.9$ & $4.7 \pm 0.9$ \\
\hline & & $88.3 \pm 0.5$ & $73.6 \pm 0.7$ & $31.9 \pm 0.7$ & $35.3 \pm 0.8$ & $21.7 \pm 0.7$ & $3.2 \pm 0.3$ \\
\hline & $\begin{array}{l}\text { Warrant officer } \\
(n=576)\end{array}$ & $92.8 \pm 0.4$ & $77.2 \pm 0.7$ & $34.2 \pm 0.8$ & $43.7 \pm 0.8$ & $12.9 \pm 0.5$ & $3.8 \pm 0.3$ \\
\hline & \multicolumn{7}{|l|}{$\begin{array}{l}\text { Junior officer } \\
(n=3,891)\end{array}$} \\
\hline & \multicolumn{7}{|l|}{$\begin{array}{l}\text { Senior officer } \\
(n=3,742)\end{array}$} \\
\hline & $\mathrm{p}$-value (chi-square) & $<0.01$ & $<0.01$ & $<0.01$ & $<0.01$ & $<0.01$ & $<0.01$ \\
\hline \multirow{5}{*}{$\begin{array}{l}\text { Occupational } \\
\text { assignment } \\
\text { group }\end{array}$} & \multirow{2}{*}{$\begin{array}{l}\text { Combat arms } \\
(\mathrm{n}=6,451)\end{array}$} & $89.0 \pm 0.4$ & $72.5 \pm 0.6$ & $27.7 \pm 0.6$ & $41.4 \pm 0.6$ & $31.9 \pm 0.6$ & $3.8 \pm 0.2$ \\
\hline & & $86.3 \pm 0.3$ & $66.2 \pm 0.5$ & $29.0 \pm 0.4$ & $42.1 \pm 0.5$ & $31.1 \pm 0.5$ & $4.2 \pm 0.2$ \\
\hline & $\begin{array}{l}\text { Combat support } \\
(n=10,424)\end{array}$ & $86.3 \pm 0.4$ & $67.6 \pm 0.5$ & $30.6 \pm 0.5$ & $42.0 \pm 0.5$ & $25.7 \pm 0.5$ & $4.6 \pm 0.2$ \\
\hline & \multicolumn{7}{|l|}{$\begin{array}{l}\text { Combat service } \\
\text { support }(n=9,132)\end{array}$} \\
\hline & $\mathrm{p}$-value (chi-square) & $<0.01$ & $<0.01$ & $<0.01$ & 0.65 & $<0.01$ & 0.04 \\
\hline \multirow{5}{*}{$\begin{array}{l}\text { Service } \\
\text { branch }\end{array}$} & Air Force $(n=9,788)$ & $86.3 \pm 0.3$ & $65.5 \pm 0.5$ & $29.7 \pm 0.5$ & $43.1 \pm 0.5$ & $28.3 \pm 0.5$ & $3.9 \pm 0.2$ \\
\hline & Army $(n=7,935)$ & $87.0 \pm 0.4$ & $69.0 \pm 0.5$ & $28.1 \pm 0.5$ & $39.8 \pm 0.5$ & $29.3 \pm 0.5$ & $4.5 \pm 0.2$ \\
\hline & \multirow{2}{*}{$\begin{array}{l}\text { Marine Corps } \\
(n=3,194)\end{array}$} & $84.8 \pm 0.6$ & $65.5 \pm 0.8$ & $23.8 \pm 0.8$ & $40.7 \pm 0.9$ & $38.8 \pm 0.9$ & $3.6 \pm 0.3$ \\
\hline & & $88.8 \pm 0.4$ & $72.8 \pm 0.6$ & $33.0 \pm 0.6$ & $43.3 \pm 0.7$ & $25.9 \pm 0.6$ & $5.0 \pm 0.3$ \\
\hline & p-value (chi-square) & $<0.01$ & $<0.01$ & $<0.01$ & $<0.01$ & $<0.01$ & $<0.01$ \\
\hline
\end{tabular}

Table 1 indicates there was little difference between men and women in aggregate consumption of any caffeinated product or coffee; however, women were much more likely to consume tea and gums/medications while men were much more likely to consume soda and energy drinks. The proportion of SMs using any caffeinated product increased with age, especially for coffee, tea, soda, and gums/medications, but use of energy drinks decreased with age. The proportion of SMs consuming any caffeinated product increased with formal educational level, especially for coffee and tea, while consumption of soda and energy drinks decreased as formal education increased; consumption of gums/medications were highest among those with some college. Among race/ethnicities, White SMs had the largest proportion consuming caffeinated products, especially for coffee, soda, and energy drinks, while Black SMs had the lowest proportion consuming these same products. As BMI increased, so did consumption of most caffeinated products, except tea, as prevalence was highest among the lowest BMI category.

For most caffeinated products, aerobic exercise duration was not related to use in any systematic way, although energy drink use increased modestly as exercise duration increased. SMs performing the most resistance training had the lowest use of any caffeinated products, especially for coffee and tea. As resistance training increased, consumption of soda decreased, and consumption of energy drinks increased. Among those who reported any weekly resistance training $(n=22,872)$, use of any caffeinated product was $87.9 \pm 0.2 \%$, compared to $80.5 \pm 0.6 \%$ among those who did not report any weekly resistance training $(n=3,808)(p<0.01)$. Smokers and former smokers had the highest use of caffeinated products among all product types except tea, where there were no significant differences among groups. Smokeless tobacco users and former users also had the highest use of caffeine for all product types except tea, where those who had never used smokeless tobacco had the highest caffeine use. Use of caffeinated products among all types increased as alcohol consumption increased. Those reporting $\geq 5$ hours/night of sleep had the highest aggregated 
caffeine and coffee use, but those reporting $<6$ hours/night had the highest use of tea, soda, energy beverages, and gums/medications.

Among both enlisted SMs and officers, as rank increased, so did aggregated use of caffeinated products, especially coffee, tea, soda, and gum/medication. For energy drinks, the trend was the opposite: as rank increased, energy drink use decreased. Enlisted soldiers were more likely to consume energy drinks than officers, and the lowest use of energy drinks was among senior officers. SMs in combat arms occupations were more likely to use any caffeinated product, especially coffee and energy drinks, while combat service support personnel had the highest use of tea and gums/medications. Navy personnel had the highest use of caffeinated products of all types, except energy drinks, where Marine Corps personnel had the highest use.

\section{Caffeine Consumption}

Table 2 provides the estimated daily caffeine consumption ( $\mathrm{mg} /$ day) among caffeine consumers by their demographic, lifestyle, and military characteristics. The average daily consumption of caffeine was $243 \mathrm{mg} / \mathrm{day}$. Coffee, tea, soda, energy drinks, and gums/medications accounted for $69 \%, 8 \%, 6 \%, 17 \%$, and $>1 \%$ of caffeine consumption, respectively. The per-capita consumption (all participants including non-consumers) was $211 \pm 1 \mathrm{mg} /$ day, with men ingesting $218 \pm 2 \mathrm{mg} /$ day and women $167 \pm 3 \mathrm{mg} /$ day. The proportion of caffeine consumers who had an overall intake $\geq 400 \mathrm{mg} /$ day was $13.8 \%$ ( $14.9 \%$ of men and $7.5 \%$ of women), and the proportion with an overall consumption $\geq 300 \mathrm{mg} /$ day was $23.7 \%$ ( $25.1 \%$ of men and $15.1 \%$ of women). The types of products ingested by the higher-quantity caffeine consumers ( $\geq 400 \mathrm{mg} / \mathrm{day}$ ) were similar to those of the entire group: coffee, teas, sodas, energy drinks, and gums/medications accounted for $68 \%, 7 \%, 5 \%, 19 \%$, and $1 \%$ of caffeine consumption, respectively. 
Table 2

Caffeine consumption (mean \pm SE mg/day) of consumers ( $\geq 1$ time/week) by demographic, lifestyle, and military characteristics

\begin{tabular}{|c|c|c|c|c|c|c|c|}
\hline Variable & Strata & $\begin{array}{l}\text { Any } \\
\text { Caffeine }\end{array}$ & Coffee & Tea & Soda & $\begin{array}{l}\text { Energy } \\
\text { Drink }\end{array}$ & $\begin{array}{l}\text { Gums \& } \\
\text { Medications }\end{array}$ \\
\hline Group & All $(n=23,175)$ & $243.1 \pm 1.5$ & $166.6 \pm 1.2$ & $18.5 \pm 0.4$ & $15.8 \pm 0.2$ & $41.0 \pm 0.6$ & $1.0 \pm 0.2$ \\
\hline \multirow[t]{3}{*}{ Gender } & Men $(n=20,040)$ & $250.7 \pm 1.7$ & $171.8 \pm 1.5$ & $17.5 \pm 0.4$ & $16.5 \pm 0.2$ & $43.9 \pm 0.7$ & $1.0 \pm 0.2$ \\
\hline & Women $(n=3.135)$ & $194.5 \pm 3.0$ & $133.8 \pm 2.5$ & $25.2 \pm 0.9$ & $11.5 \pm 0.5$ & $23.7 \pm 1.3$ & $1.2 \pm 0.3$ \\
\hline & p-value (ANOVA) & $<0.01$ & $<0.01$ & $<0.01$ & $<0.01$ & $<0.01$ & 0.60 \\
\hline \multirow[t]{6}{*}{ Age } & \multirow{2}{*}{$\begin{array}{l}\text { 18-24 years } \\
(n=3,615)\end{array}$} & $203.6 \pm 4.6$ & $108.9 \pm 2.7$ & $19.5 \pm 1.0$ & $17.4 \pm 0.7$ & $56.0 \pm 2.1$ & $1.8 \pm 0.6$ \\
\hline & & $226.1 \pm 3.1$ & $147.7 \pm 2.4$ & $16.5 \pm 0.7$ & $13.1 \pm 0.4$ & $47.7 \pm 1.5$ & $1.0 \pm 0.2$ \\
\hline & $\begin{array}{l}25-29 \text { years } \\
(n=4,746)\end{array}$ & $254.7 \pm 2.2$ & $179.2 \pm 1.8$ & $17.3 \pm 0.5$ & $15.5 \pm 0.3$ & $42.0 \pm 0.9$ & $0.7 \pm 0.2$ \\
\hline & $\begin{array}{l}30-39 \text { years } \\
(n=9,875)\end{array}$ & $264.8 \pm 3.4$ & $201.6 \pm 2.8$ & $22.0 \pm 0.8$ & $18.0 \pm 0.5$ & $22.1 \pm 1.1$ & $1.1 \pm 0.4$ \\
\hline & $\begin{array}{l}\geq 40 \text { years } \\
(n=4,821)\end{array}$ & & & & & & \\
\hline & $\begin{array}{l}\text { p-value } \\
\text { (ANOVA/trend) }\end{array}$ & $<0.01 /<0.01$ & $<0.01 /<0.01$ & $<0.01 / 0.03$ & $<0.01 / 0.07$ & $<0.01 / 0.01$ & $0.15 / 0.14$ \\
\hline \multirow[t]{5}{*}{ Education } & \multirow{2}{*}{$\begin{array}{l}\text { Some high } \\
\text { school/high school } \\
\text { graduate }(n=3,099)\end{array}$} & $242.3 \pm 4.8$ & $129.3 \pm 1.9$ & $20.4 \pm 1.3$ & $20.9 \pm 0.8$ & $69.7 \pm 2.5$ & $1.9 \pm 0.4$ \\
\hline & & $243.3 \pm 2.4$ & $159.5 \pm 1.7$ & $18.1 \pm 0.5$ & $16.5 \pm 0.4$ & $48.3 \pm 1.0$ & $1.0 \pm 0.3$ \\
\hline & $\begin{array}{l}\text { Some } \\
\text { college/Associate's } \\
\text { degree }(n=9,836)\end{array}$ & $243.0 \pm 2.1$ & $184.8 \pm 1.2$ & $18.4 \pm 0.5$ & $13.6 \pm 0.3$ & $25.4 \pm 0.8$ & $0.8 \pm 02$ \\
\hline & $\begin{array}{l}\text { Bachelor's/Graduate } \\
\text { degree }(n=10,234)\end{array}$ & & & & & & \\
\hline & $\begin{array}{l}\text { p-value } \\
\text { (ANOVA/trend) }\end{array}$ & $0.98 / 0.88$ & $<0.01 /<0.01$ & $0.10 / 0.06$ & $<0.01 /<0.01$ & $<0.01 /<0.01$ & $0.06 / 0.02$ \\
\hline \multirow{5}{*}{$\begin{array}{l}\text { Race / } \\
\text { Ethnicity }\end{array}$} & White $(n=14,827)$ & $263.5 \pm 1.9$ & $184.8 \pm 1.5$ & $18.0 \pm 0.4$ & $17.3 \pm 0.3$ & $42.5 \pm 0.8$ & $0.8 \pm 0.2$ \\
\hline & Hispanic $(n=3,530)$ & $227.7 \pm 4.1$ & $151.8 \pm 3.0$ & $15.9 \pm 0.9$ & $13.5 \pm 0.6$ & $45.3 \pm 2.0$ & $1.1 \pm 0.3$ \\
\hline & Back $(n=2,140)$ & $158.8 \pm 3.8$ & $90.9 \pm 2.7$ & $21.5 \pm 1.0$ & $13.1 \pm 0.7$ & $31.1 \pm 1.9$ & $2.2 \pm 0.5$ \\
\hline & Other $(n=2,678)$ & $217.5 \pm 3.9$ & $146.3 \pm 3.1$ & $22.0 \pm 1.1$ & $12.6 \pm 0.6$ & $35.3 \pm 1.6$ & $1.3 \pm 0.3$ \\
\hline & p-value (ANOVA) & $<0.01$ & $<0.01$ & $<0.01$ & $<0.01$ & $<0.01$ & 0.06 \\
\hline \multirow{5}{*}{$\begin{array}{l}\text { Body mass } \\
\text { index }\end{array}$} & \multirow{2}{*}{$\begin{array}{l}<25.0 \mathrm{~kg} / \mathrm{m}^{2} \\
(\mathrm{n}=6,629)\end{array}$} & $215.7 \pm 3.0$ & $145.4 \pm 2.0$ & $19.2 \pm 0.7$ & $15.1 \pm 0.4$ & $34.3 \pm 1.2$ & $1.6 \pm 0.5$ \\
\hline & & $251.2 \pm 2.0$ & $175.7 \pm 1.7$ & $17.7 \pm 0.5$ & $15.2 \pm 0.3$ & $42.0 \pm 0.9$ & $0.7 \pm 0.1$ \\
\hline & $\begin{array}{l}25.0-29.9 \mathrm{~kg} / \mathrm{m}^{2} \\
(\mathrm{n}=12,163)\end{array}$ & $260.5 \pm 3.5$ & $172.3 \pm 2.9$ & $19.2 \pm 0.9$ & $19.1 \pm 0.6$ & $48.8 \pm 1.5$ & $1.1 \pm 0.3$ \\
\hline & $\underset{(n=3,956)}{\geq 30.0 \mathrm{~kg} / \mathrm{m}^{2}}$ & & & & & & \\
\hline & $\begin{array}{l}\text { p-value } \\
\text { (ANOVA/trend) }\end{array}$ & $<0.01 /<0.01$ & $<0.01 /<0.01$ & $0.09 / 0.97$ & $<0.01 /<0.01$ & $<0.01 /<0.01$ & $0.03 / 0.28$ \\
\hline
\end{tabular}




\begin{tabular}{|c|c|c|c|c|c|c|c|}
\hline Variable & Strata & $\begin{array}{l}\text { Any } \\
\text { Caffeine }\end{array}$ & Coffee & Tea & Soda & $\begin{array}{l}\text { Energy } \\
\text { Drink }\end{array}$ & $\begin{array}{l}\text { Gums \& } \\
\text { Medications }\end{array}$ \\
\hline \multirow{6}{*}{$\begin{array}{l}\text { Aerobic } \\
\text { exercise } \\
\text { weekly } \\
\text { duration }\end{array}$} & $\begin{array}{l}<90 \mathrm{~min} / \mathrm{wk} \\
(\mathrm{n}=6,120)\end{array}$ & $235.7 \pm 3.0$ & $161.0 \pm 2.2$ & $17.8 \pm 0.7$ & $16.5 \pm 0.4$ & $39.2 \pm 1.2$ & $1.1 \pm 0.4$ \\
\hline & & $236.2 \pm 2.4$ & $165.3 \pm 2.0$ & $17.6 \pm 0.6$ & $16.5 \pm 0.4$ & $36.3 \pm 1.0$ & $0.6 \pm 0.1$ \\
\hline & $(n=6,560)$ & $249.1 \pm 3.1$ & $173.9 \pm 2.5$ & $17.5 \pm 0.7$ & $15.1 \pm 0.4$ & $42.0 \pm 1.4$ & $0.6 \pm 0.1$ \\
\hline & $\begin{array}{l}181-300 \mathrm{~min} / \mathrm{wk} \\
(\mathrm{n}=5,218)\end{array}$ & $254.0 \pm 3.9$ & $167.7 \pm 2.8$ & $21.5 \pm 0.7$ & $14.9 \pm 0.5$ & $48.1 \pm 1.6$ & $1.8 \pm 0.5$ \\
\hline & $\begin{array}{l}>300 \mathrm{~min} / \mathrm{wk} \\
(\mathrm{n}=5,277)\end{array}$ & & & & & & \\
\hline & $\begin{array}{l}\text { p-value } \\
\text { (ANOVA/trend) }\end{array}$ & $<0.01 /<0.01$ & $<0.01 / 0.01$ & $<0.01 /<0.01$ & $0.01 /<0.01$ & $<0.01 /<0.01$ & $0.02 / 0.15$ \\
\hline \multirow{6}{*}{$\begin{array}{l}\text { Resistance } \\
\text { training } \\
\text { weekly } \\
\text { duration }\end{array}$} & $\leq 45 \mathrm{~min} / \mathrm{wk}(\mathrm{n}=$ & $250.8 \pm 3.0$ & $172.8 \pm 2.3$ & $21.1 \pm 0.7$ & $20.6 \pm 0.5$ & $35.3 \pm 1.2$ & $1.0 \pm 0.3$ \\
\hline & & $239.3 \pm 2.6$ & $171.2 \pm 2.3$ & $18.3 \pm 0.7$ & $15.8 \pm 0.4$ & $33.5 \pm 1.0$ & $0.6 \pm 0.1$ \\
\hline & $=5,636)$ & $238.5 \pm 2.7$ & $166.8 \pm 2.2$ & $16.3 \pm 0.6$ & $13.5 \pm 0.4$ & $41.2 \pm 1.2$ & $0.8 \pm 0.2$ \\
\hline & $\begin{array}{l}136-300 \mathrm{~min} / \mathrm{wk}(\mathrm{n} \\
=5,859)\end{array}$ & $242.3 \pm 3.9$ & $153.2 \pm 2.7$ & $18.0 \pm 0.8$ & $12.3 \pm 0.4$ & $56.9 \pm 1.6$ & $1.8 \pm 0.5$ \\
\hline & $\begin{array}{l}\geq 301 \mathrm{~min} / \mathrm{wk}(\mathrm{n}= \\
5,030)\end{array}$ & & & & & & \\
\hline & $\begin{array}{l}\text { p-value } \\
\text { (ANOVA/trend) }\end{array}$ & $0.01 / 0.06$ & $<0.01 / 0.01$ & $<0.01 /<0.01$ & $<0.01 /<0.01$ & $<0.01 /<0.01$ & $0.05 / 0.08$ \\
\hline \multirow[t]{4}{*}{ Smoking } & Never $(n=14,177)$ & $215.5 \pm 1.8$ & $148.2 \pm 1.4$ & $18.7 \pm 0.4$ & $14.6 \pm 0.3$ & $33.0 \pm 0.7$ & $1.0 \pm 0.2$ \\
\hline & Former user & $278.8 \pm 3.4$ & $198.4 \pm 2.8$ & $17.5 \pm 0.8$ & $15.6 \pm 0.5$ & $46.3 \pm 1.4$ & $0.9 \pm 0.2$ \\
\hline & $\begin{array}{l}\text { Current user } \\
(n=4,222)\end{array}$ & $298.7 \pm 4.4$ & $195.3 \pm 3.2$ & $19.1 \pm 0.9$ & $20.3 \pm 0.6$ & $62.7 \pm 1.9$ & $1.3 \pm 0.5$ \\
\hline & p-value (ANOVA) & $<0.01$ & $<0.01$ & 0.34 & $<0.01$ & $<0.01$ & 0.70 \\
\hline \multirow{4}{*}{$\begin{array}{l}\text { Smokeless } \\
\text { tobacco use }\end{array}$} & Never $(n=17,597)$ & $226.8 \pm 1.6$ & $155.8 \pm 1.3$ & $18.7 \pm 0.4$ & $15.6 \pm 0.3$ & $35.8 \pm 0.7$ & $1.0 \pm 0.2$ \\
\hline & Former user & $294.5 \pm 4.7$ & $213.9 \pm 4.1$ & $16.5 \pm 1.2$ & $14.9 \pm 0.7$ & $48.6 \pm 2.1$ & $0.7 \pm 0.3$ \\
\hline & $\begin{array}{l}\text { Current user } \\
(n=2,938)\end{array}$ & $301.5 \pm 5.4$ & $195.9 \pm 3.6$ & $18.9 \pm 1.3$ & $18.2 \pm 0.7$ & $66.9 \pm 2.4$ & $1.6 \pm 0.7$ \\
\hline & p-value (ANOVA) & $<0.01$ & $<0.01$ & 0.20 & $<0.01$ & $<0.01$ & 0.32 \\
\hline \multirow{6}{*}{$\begin{array}{l}\text { Alcohol } \\
\text { intake }\end{array}$} & $0 \mathrm{ml} / \mathrm{wk}(\mathrm{n}=6,175)$ & $218.9 \pm 3.1$ & $137.6 \pm 2.4$ & $19.5 \pm 0.7$ & $17.6 \pm 0.5$ & $39.5 \pm 1.3$ & $1.1 \pm 0.3$ \\
\hline & $0.23-24.85 \mathrm{ml} / \mathrm{wk}$ & $214.8 \pm 2.6$ & $149.6 \pm 2.2$ & $18.2 \pm 0.6$ & $14.6 \pm 0.4$ & $29.8 \pm 1.1$ & $0.5 \pm 0.1$ \\
\hline & & $239.0 \pm 2.6$ & $169.2 \pm 2.2$ & $17.2 \pm 0.7$ & $14.2 \pm 0.4$ & $35.3 \pm 1.1$ & $0.7 \pm 0.2$ \\
\hline & $(n=5,696)$ & $299.2 \pm 3.6$ & $211.1 \pm 2.6$ & $19.3 \pm 0.8$ & $16.7 \pm 0.5$ & $45.8 \pm 1.5$ & $1.8 \pm 0.5$ \\
\hline & $\underset{(\mathrm{n}=5,817)}{\geq 71.70 \mathrm{ml} / \mathrm{wk}}$ & & & & & & \\
\hline & $\begin{array}{l}\text { p-value } \\
\text { (ANOVA/trend) }\end{array}$ & $<0.01 /<0.01$ & $<0.01 /<0.01$ & $0.05 / 0.60$ & $<0.01 / 0.17$ & $<0.01 /<0.01$ & $0.02 / 0.13$ \\
\hline
\end{tabular}




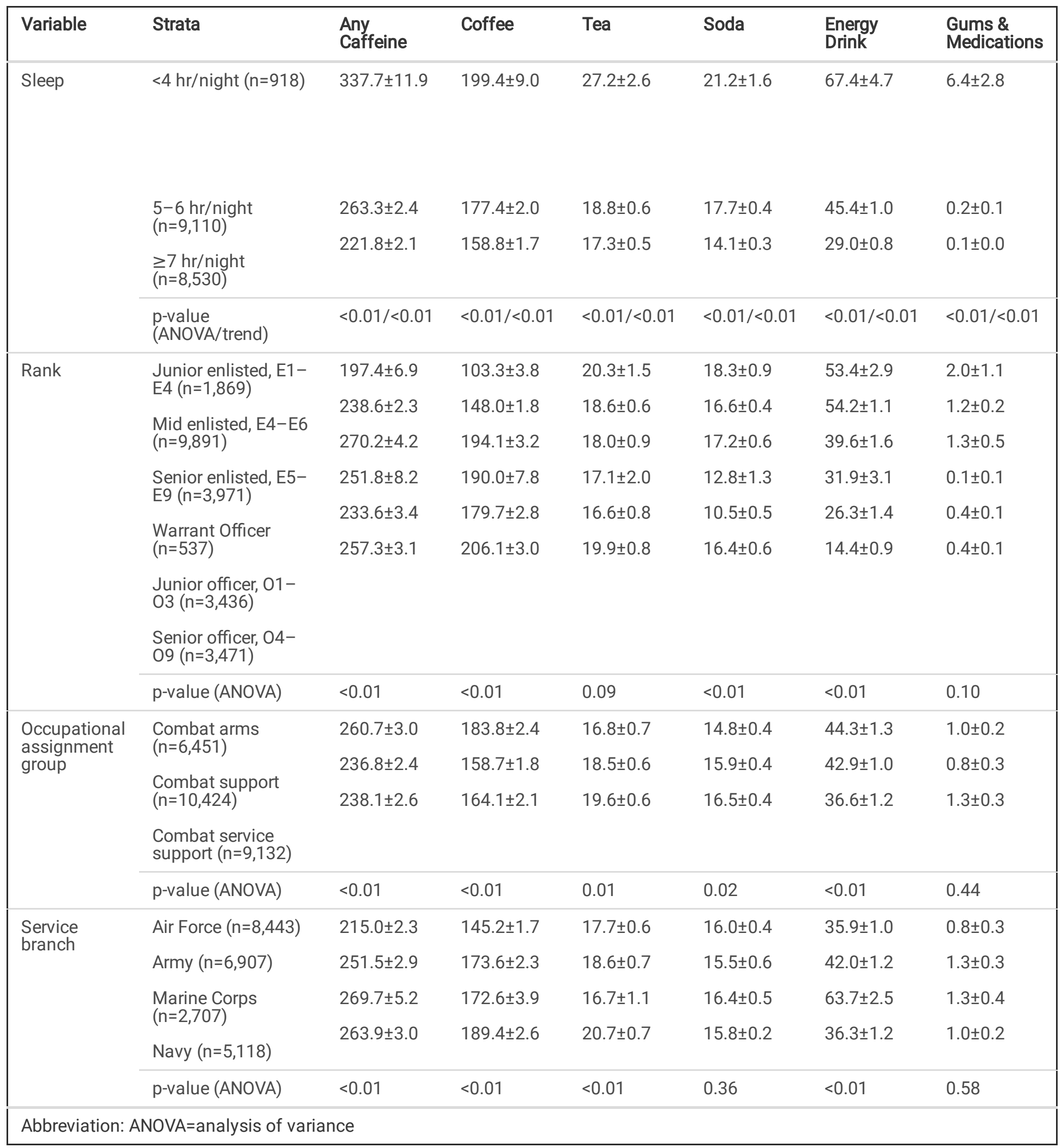

Men consumed more total caffeine than women due to a greater consumption from coffee, soda, and energy drinks; women consumed more caffeine from tea. When total caffeine consumption was determined on a body weight basis, consumption was similar among male and female consumers ( 2.93 vs $2.85 \mathrm{mg} /$ day $/ \mathrm{kg}$, men and women, respectively, $\mathrm{p}=0.12$ ). Caffeine consumption increased with age, largely accounted for by the increase from coffee, while caffeine consumption from energy drinks decreased with age. Caffeine consumed from tea and soda was greatest in the youngest and oldest age groups. Total caffeine consumption differed little by formal educational level, but caffeine from coffee increased with more formal education, while caffeine from soda, energy drinks, and gums/medications decreased with more formal education. White and Hispanic SMs consumed the most total caffeine, accounted for largely by coffee, soda, and energy beverages, while Black SMs consumed the least total caffeine and had the least 
caffeine consumption from coffee, soda, and energy beverages. As BMI increased, so did total caffeine consumption, especially from coffee, soda, and energy drinks; caffeine from gums/medications was highest among the lowest BMI group.

As the amount of aerobic exercise increased, so did total caffeine consumption, accounted for largely by caffeine from coffee and energy drinks. Caffeine from soda decreased as aerobic exercise increased; caffeine from tea and gums/medications was highest in the group performing the most aerobic exercise. As the amount of resistance training increased, caffeine from coffee, tea, and soda tended to decrease, while caffeine from energy drinks increased. Among smokers and smokeless tobacco users, the pattern of caffeine consumption was similar: current and former users had the highest total caffeine consumption, accounted for largely by consumption from coffee, soda, and energy drinks. As alcohol intake increased, so did the total consumption of caffeine, accounted for by caffeine from coffee. Caffeine from tea, soda, energy drinks, and gums/medications was highest among non-alcohol users and those in the highest alcohol level. Consumption of caffeine from all sources increased as the amount of sleep decreased. The average \pm standard deviation for self-reported sleep was $6.3 \pm 1.4$ hours.

Consumption of total caffeine and caffeine from coffee increased with rank among enlisted personnel and officers, while consumption from energy drinks decreased with rank among enlisted and officers. Caffeine from soda decreased with rank among enlisted SMs but increased with rank among officers. SMs employed in combat arms occupations had the highest total consumption of caffeine and consumption from coffee and energy drinks, while those in combat service support occupations had the highest consumption from tea and soda. Marine Corps and Navy personnel had the highest total consumption of caffeine. Caffeine from coffee and tea was highest among Navy personnel, while caffeine from energy drinks was highest among Marine Corps personnel. Air Force personnel had the lowest total caffeine consumption and the lowest consumption from coffee and energy drinks.

\section{ICharacteristics Independently Associated with Caffeine Prevalence}

Table 3 provides results of the multivariable logistic regression examining factors associated with the use of caffeinated products $\geq 1$ time per week. The results are for six full models with all characteristics entered. There were $91 \%(n=24,324)$ of SMs with complete data on all variables that were included in each model. 
Table 3

Characteristics associated with prevalence ( $\geq 1$ time/week) of specific caffeine products among military personnel. Multivariable logistic regression, data are odds ratios with $95 \%$ confidence intervals in parenthesis

\begin{tabular}{|c|c|c|c|c|c|c|c|}
\hline \multirow[t]{2}{*}{ Variable } & \multirow[t]{2}{*}{ Strata } & \multicolumn{6}{|c|}{ Caffeine Products Consumed $\geq 1$ Time/Week [Odds ratio ( $95 \%$ confidence interval)] } \\
\hline & & $\begin{array}{l}\text { Any Caffeine } \\
\text { Product } \\
\text { (Model 1) }\end{array}$ & $\begin{array}{l}\text { Coffee } \\
\text { (Model 2) }\end{array}$ & $\begin{array}{l}\text { Tea * } \\
\text { (Model 3) }\end{array}$ & $\begin{array}{l}\text { Soda } \\
\text { (Model 4) }\end{array}$ & $\begin{array}{l}\text { Energy } \\
\text { Drink } \\
\text { (Model 5) }\end{array}$ & $\begin{array}{l}\text { Gum or } \\
\text { Medication } \\
\text { (Model 6) }\end{array}$ \\
\hline \multirow[t]{2}{*}{ Gender } & Men & 1.00 & 1.00 & 1.00 & 1.00 & 1.00 & 1.00 \\
\hline & Women & $\begin{array}{l}1.52(1.34- \\
1.72)\end{array}$ & $\begin{array}{l}1.43 \\
(1.31- \\
1.56)\end{array}$ & $\begin{array}{l}2.14 \\
(1.97- \\
2.32)\end{array}$ & $\begin{array}{l}0.71 \\
(0.66- \\
0.77)\end{array}$ & $\begin{array}{l}0.64 \\
(0.58- \\
0.71)\end{array}$ & $\begin{array}{l}2.10(1.78- \\
2.49)\end{array}$ \\
\hline \multirow[t]{5}{*}{ Age } & $18-24$ years & 1.00 & 1.00 & 1.00 & 1.00 & 1.00 & 1.00 \\
\hline & $\begin{array}{l}25-29 \text { years } \\
30-39 \text { years }\end{array}$ & $\begin{array}{l}1.25(1.10- \\
1.44)\end{array}$ & $\begin{array}{l}1.34 \\
(1.21- \\
1.49)\end{array}$ & $\begin{array}{l}1.03 \\
(0.92- \\
1.14)\end{array}$ & $\begin{array}{l}0.93 \\
(0.84- \\
1.02)\end{array}$ & $\begin{array}{l}1.06 \\
(0.96- \\
1.17)\end{array}$ & $\begin{array}{l}0.99(0.77- \\
1.26)\end{array}$ \\
\hline & \multirow[t]{3}{*}{$\geq 40$ years } & $\begin{array}{l}1.69(1.46- \\
1.95)\end{array}$ & \multirow{2}{*}{$\begin{array}{l}1.59 \\
(1.43- \\
1.76)\end{array}$} & \multirow{2}{*}{$\begin{array}{l}1.08 \\
(0.97- \\
1.21)\end{array}$} & \multirow{2}{*}{$\begin{array}{l}1.05 \\
(0.95- \\
1.15)\end{array}$} & \multirow{2}{*}{$\begin{array}{l}1.01 \\
(0.91- \\
1.13)\end{array}$} & \multirow{3}{*}{$\begin{array}{l}1.34(1.05- \\
1.70) \\
1.54(1.15- \\
2.05)\end{array}$} \\
\hline & & \multirow{2}{*}{$\begin{array}{l}1.95(1.61- \\
2.38)\end{array}$} & & & & & \\
\hline & & & $\begin{array}{l}1.79 \\
(1.56- \\
2.05)\end{array}$ & $\begin{array}{l}1.36 \\
(1.19- \\
1.55)\end{array}$ & $\begin{array}{l}1.08 \\
(0.96- \\
1.23)\end{array}$ & $\begin{array}{l}0.61 \\
(0.53- \\
0.70)\end{array}$ & \\
\hline \multirow[t]{4}{*}{ Education } & Some HS/HS grad & 1.00 & 1.00 & 1.00 & 1.00 & 1.00 & 1.00 \\
\hline & \multirow{3}{*}{$\begin{array}{l}\text { Some college } \\
\text { College degree }\end{array}$} & $\begin{array}{l}1.07(0.94- \\
1.22)\end{array}$ & \multirow{2}{*}{$\begin{array}{l}1.27 \\
(1.16- \\
1.40)\end{array}$} & \multirow{2}{*}{$\begin{array}{l}1.13 \\
(1.02- \\
1.25)\end{array}$} & \multirow{2}{*}{$\begin{array}{l}0.84 \\
(0.77- \\
0.92)\end{array}$} & \multirow{2}{*}{$\begin{array}{l}0.93 \\
(0.85- \\
1.02)\end{array}$} & $\begin{array}{l}0.93(0.75- \\
1.15)\end{array}$ \\
\hline & & \multirow{2}{*}{$\begin{array}{l}1.09(0.92- \\
1.30)\end{array}$} & & & & & \multirow{2}{*}{$\begin{array}{l}0.80(0.62- \\
1.04)\end{array}$} \\
\hline & & & $\begin{array}{l}1.38 \\
(1.22- \\
1.56)\end{array}$ & $\begin{array}{l}1.16 \\
(1.03- \\
1.31)\end{array}$ & $\begin{array}{l}0.74 \\
(0.66- \\
0.83)\end{array}$ & $\begin{array}{l}0.75 \\
(0.67- \\
0.85)\end{array}$ & \\
\hline \multirow[t]{6}{*}{ Race / Ethnicity } & White & 1.00 & 1.00 & 1.00 & 1.00 & 1.00 & 1.00 \\
\hline & & \multirow{3}{*}{$\begin{array}{l}0.64(0.57- \\
0.72) \\
0.27(0.24- \\
0.31)\end{array}$} & \multirow{2}{*}{$\begin{array}{l}0.87 \\
(0.80- \\
0.95)\end{array}$} & \multirow{2}{*}{$\begin{array}{l}0.88 \\
(0.81- \\
0.96)\end{array}$} & \multirow{2}{*}{$\begin{array}{l}0.86 \\
(0.79- \\
0.92)\end{array}$} & \multirow{2}{*}{$\begin{array}{l}0.80 \\
(0.74- \\
0.87)\end{array}$} & $\begin{array}{l}0.75(0.62- \\
0.91)\end{array}$ \\
\hline & Back & & & & & & $0.84(0.68-$ \\
\hline & \multirow[t]{3}{*}{ Other } & & 0.36 & 1.08 & 0.71 & 0.47 & $1.03)$ \\
\hline & & $0.65(0.58-$ & $0.40)$ & $1.19)$ & $0.77)$ & $0.53)$ & $0.74(0.59-$ \\
\hline & & & $\begin{array}{l}0.90 \\
(0.82- \\
0.99)\end{array}$ & $\begin{array}{l}1.35 \\
(1.23- \\
1.47)\end{array}$ & $\begin{array}{l}0.88 \\
(0.81- \\
0.96)\end{array}$ & $\begin{array}{l}0.78 \\
(0.72- \\
0.87)\end{array}$ & \\
\hline Body mass index & $<25.0 \mathrm{~kg} / \mathrm{m}^{2}$ & 1.00 & 1.00 & 1.00 & 1.00 & 1.00 & 1.00 \\
\hline & $25.0-29.9 \mathrm{~kg} / \mathrm{m}^{2}$ & $\begin{array}{l}1.19(1.08- \\
1.30)\end{array}$ & $\begin{array}{l}1.08 \\
(1.01-\end{array}$ & $\begin{array}{l}0.96 \\
(0.90-\end{array}$ & $\begin{array}{l}1.09 \\
(1.02-\end{array}$ & $\begin{array}{l}1.29 \\
(1.20-\end{array}$ & $\begin{array}{l}1.20(1.02- \\
1.40)\end{array}$ \\
\hline & $\geq 30.0 \mathrm{~kg} / \mathrm{m}^{2}$ & 1.46 (1.27- & & & & & $1.61(1.33-$ \\
\hline & & $1.67)$ & $\begin{array}{l}1.08 \\
(0.99- \\
1.19)\end{array}$ & $\begin{array}{l}1.00 \\
(0.91- \\
1.09)\end{array}$ & $\begin{array}{l}1.43 \\
(1.32- \\
1.56)\end{array}$ & $\begin{array}{l}1.58 \\
(1.44- \\
1.73)\end{array}$ & $1.95)$ \\
\hline
\end{tabular}




\begin{tabular}{|c|c|c|c|c|c|c|c|}
\hline \multirow[t]{2}{*}{ Variable } & \multirow[t]{2}{*}{ Strata } & \multicolumn{6}{|c|}{ Caffeine Products Consumed $\geq 1$ Time/Week [Odds ratio ( $95 \%$ confidence interval)] } \\
\hline & & $\begin{array}{l}\text { Any Caffeine } \\
\text { Product } \\
\text { (Model 1) }\end{array}$ & $\begin{array}{l}\text { Coffee } \\
\text { (Model 2) }\end{array}$ & $\begin{array}{l}\text { Tea * } \\
\text { (Model 3) }\end{array}$ & $\begin{array}{l}\text { Soda } \\
\text { (Model 4) }\end{array}$ & $\begin{array}{l}\text { Energy } \\
\text { Drink } \\
\text { (Model 5) }\end{array}$ & $\begin{array}{l}\text { Gum or } \\
\text { Medication } \\
\text { (Model 6) }\end{array}$ \\
\hline \multirow{6}{*}{$\begin{array}{l}\text { Aerobic exercise } \\
\text { duration }\end{array}$} & $<90 \mathrm{~min} / \mathrm{wk}$ & 1.00 & 1.00 & 1.00 & 1.00 & 1.00 & 1.00 \\
\hline & \multirow{5}{*}{$\begin{array}{l}90-180 \mathrm{~min} / \mathrm{wk} \\
181-300 \mathrm{~min} / \mathrm{wk} \\
>300 \mathrm{~min} / \mathrm{wk}\end{array}$} & \multirow{3}{*}{$\begin{array}{l}1.11(0.99- \\
1.25) \\
1.07(0.95- \\
1.22)\end{array}$} & \multirow{2}{*}{$\begin{array}{l}1.07 \\
(0.99- \\
1.16)\end{array}$} & \multirow{2}{*}{$\begin{array}{l}1.12 \\
(1.04- \\
1.21)\end{array}$} & \multirow{2}{*}{$\begin{array}{l}1.05 \\
(0.98- \\
1.13)\end{array}$} & \multirow{2}{*}{$\begin{array}{l}1.01 \\
(0.93- \\
1.09)\end{array}$} & $\begin{array}{l}0.93(0.78- \\
1.11)\end{array}$ \\
\hline & & & & & & & \multirow{4}{*}{$\begin{array}{l}0.86(0.71- \\
1.03) \\
1.07(0.88- \\
1.29)\end{array}$} \\
\hline & & & \multirow{2}{*}{$\begin{array}{l}1.05 \\
(0.96- \\
1.14)\end{array}$} & \multirow{2}{*}{$\begin{array}{l}1.14 \\
(1.05- \\
1.24)\end{array}$} & \multirow{2}{*}{$\begin{array}{l}1.01 \\
(0.93- \\
1.09)\end{array}$} & \multirow{2}{*}{$\begin{array}{l}1.00 \\
(0.92- \\
1.09)\end{array}$} & \\
\hline & & \multirow[t]{2}{*}{$\begin{array}{l}0.95(0.84- \\
1.08)\end{array}$} & & & & & \\
\hline & & & $\begin{array}{l}1.00 \\
(0.91- \\
1.09)\end{array}$ & $\begin{array}{l}1.22 \\
(1.12- \\
1.33)\end{array}$ & $\begin{array}{l}0.93 \\
(0.86- \\
1.01)\end{array}$ & $\begin{array}{l}0.88 \\
(0.81- \\
0.96)\end{array}$ & \\
\hline \multirow{6}{*}{$\begin{array}{l}\text { Resistance } \\
\text { training } \\
\text { duration }\end{array}$} & $\leq 45 \mathrm{~min} / \mathrm{wk}$ & 1.00 & 1.00 & 1.00 & 1.00 & 1.00 & 1.00 \\
\hline & \multirow{2}{*}{$\begin{array}{l}46-135 \mathrm{~min} / \mathrm{wk} \\
136-300 \mathrm{~min} / \mathrm{wk}\end{array}$} & \multirow{2}{*}{$\begin{array}{l}1.08(0.96- \\
1.23)\end{array}$} & \multirow{2}{*}{$\begin{array}{l}1.16 \\
(1.07- \\
1.26)\end{array}$} & \multirow{2}{*}{$\begin{array}{l}0.99 \\
(0.92- \\
1.07)\end{array}$} & \multirow{2}{*}{$\begin{array}{l}0.85 \\
(0.79- \\
0.91)\end{array}$} & \multirow{2}{*}{$\begin{array}{l}1.03 \\
(0.94- \\
1.12)\end{array}$} & \multirow{2}{*}{$\begin{array}{l}1.00(0.84- \\
1.19)\end{array}$} \\
\hline & & & & & & & \\
\hline & \multirow[t]{3}{*}{$\geq 301 \mathrm{~min} / \mathrm{wk}$} & $\begin{array}{l}1.06(0.93- \\
1.20)\end{array}$ & \multirow{2}{*}{$\begin{array}{l}1.22 \\
(1.12- \\
1.32)\end{array}$} & \multirow{2}{*}{$\begin{array}{l}0.93 \\
(0.86- \\
1.01)\end{array}$} & \multirow{2}{*}{$\begin{array}{l}0.63 \\
(0.59- \\
0.68)\end{array}$} & 1.11 & \multirow{3}{*}{$\begin{array}{l}0.97(0.81- \\
1.15) \\
0.88(0.72- \\
1.08)\end{array}$} \\
\hline & & $\begin{array}{l}0.75(0.66- \\
0.85)\end{array}$ & & & & $1.21)$ & \\
\hline & & & $\begin{array}{l}0.98 \\
(0.89- \\
1.07)\end{array}$ & $\begin{array}{l}0.74 \\
(0.68- \\
0.81)\end{array}$ & $\begin{array}{l}0.49 \\
(0.45- \\
0.53)\end{array}$ & $\begin{array}{l}1.30 \\
(1.19- \\
1.43)\end{array}$ & \\
\hline Smoking & Never & 1.00 & 1.00 & 1.00 & 1.00 & 1.00 & 1.00 \\
\hline & Former user & $\begin{array}{l}1.77(1.54- \\
2.05)\end{array}$ & $\begin{array}{l}1.74 \\
(1.59-\end{array}$ & $\begin{array}{l}1.05 \\
(0.97-\end{array}$ & $\begin{array}{l}1.07 \\
(1.00-\end{array}$ & $\begin{array}{l}1.16 \\
(1.07-\end{array}$ & $\begin{array}{l}1.17(0.98- \\
1.38)\end{array}$ \\
\hline & Current user & & 1.90) & $1.14)$ & $1.16)$ & $1.26)$ & \\
\hline & & $2.31)$ & $\begin{array}{l}1.68 \\
(1.53- \\
1.83)\end{array}$ & $\begin{array}{l}1.09 \\
(1.00- \\
1.18)\end{array}$ & $\begin{array}{l}1.45 \\
(1.34- \\
1.56)\end{array}$ & $\begin{array}{l}1.57 \\
(1.45- \\
1.70)\end{array}$ & $1.35)$ \\
\hline Smokeless & Never & 1.00 & 1.00 & 1.00 & 1.00 & 1.00 & 1.00 \\
\hline & Former user & $\begin{array}{l}1.88(1.48- \\
2.39)\end{array}$ & $\begin{array}{l}1.57 \\
(1.37-\end{array}$ & $\begin{array}{l}0.88 \\
(0.78-\end{array}$ & $\begin{array}{l}0.90 \\
(0.82-\end{array}$ & $\begin{array}{l}1.20 \\
(1.08-\end{array}$ & $\begin{array}{l}1.04(0.82- \\
1.32)\end{array}$ \\
\hline & Current user & $1.58(1.32-$ & 1.79) & & $1.00)$ & 1.33) & \\
\hline & & 1.88) & $\begin{array}{l}1.44 \\
(1.30- \\
1.60)\end{array}$ & $\begin{array}{l}0.91 \\
(0.83- \\
0.99)\end{array}$ & $\begin{array}{l}1.07 \\
(0.98- \\
1.16)\end{array}$ & $\begin{array}{l}1.52 \\
(1.40- \\
1.66)\end{array}$ & $1.21)$ \\
\hline Alcohol intake & $0 \mathrm{ml} / \mathrm{wk}$ & 1.00 & 1.00 & 1.00 & 1.00 & 1.00 & 1.00 \\
\hline & $0.23-24.85 \mathrm{ml} / \mathrm{wk}$ & $\begin{array}{l}2.16(1.94- \\
2.40)\end{array}$ & $\begin{array}{l}1.85 \\
(1.71-\end{array}$ & $\begin{array}{l}1.38 \\
(1.27-\end{array}$ & $\begin{array}{l}1.19 \\
(1.10-\end{array}$ & $\begin{array}{l}1.08 \\
0.99-\end{array}$ & $\begin{array}{l}0.98(0.81- \\
1.17)\end{array}$ \\
\hline & $24.86-71.69 \mathrm{ml} / \mathrm{wk}$ & & 1.99) & $1.49)$ & $1.28)$ & $1.17)$ & \\
\hline & & $\begin{array}{l}3.84) \\
3.00-\end{array}$ & 2.36 & $\begin{array}{l}1.51 \\
139-\end{array}$ & 1.39 & 1.39 & $1.38)^{1.15(0.90-}$ \\
\hline & & $\begin{array}{l}5.15(4.44- \\
5.98)\end{array}$ & $2.56)$ & $1.64)$ & $1.49)$ & $1.51)$ & $1.36(1.14-$ \\
\hline & & & $\begin{array}{l}3.10 \\
(2.84- \\
3.38)\end{array}$ & $\begin{array}{l}1.68 \\
(1.54- \\
1.82)\end{array}$ & $\begin{array}{l}1.50 \\
(1.39- \\
1.62)\end{array}$ & $\begin{array}{l}1.76 \\
(1.62- \\
1.92)\end{array}$ & \\
\hline
\end{tabular}




\begin{tabular}{|c|c|c|c|c|c|c|c|}
\hline \multirow[t]{2}{*}{ Variable } & \multirow[t]{2}{*}{ Strata } & \multicolumn{6}{|c|}{ Caffeine Products Consumed $\geq 1$ Time/Week [Odds ratio ( $95 \%$ confidence interval)] } \\
\hline & & $\begin{array}{l}\text { Any Caffeine } \\
\text { Product } \\
\text { (Model 1) }\end{array}$ & $\begin{array}{l}\text { Coffee } \\
\text { (Model 2) }\end{array}$ & $\begin{array}{l}\text { Tea * } \\
\text { (Model 3) }\end{array}$ & $\begin{array}{l}\text { Soda } \\
\text { (Model 4) }\end{array}$ & $\begin{array}{l}\text { Energy } \\
\text { Drink } \\
\text { (Model 5) }\end{array}$ & $\begin{array}{l}\text { Gum or } \\
\text { Medication } \\
\text { (Model 6) }\end{array}$ \\
\hline \multirow[t]{7}{*}{ Rank } & $\begin{array}{l}\text { Junior enlisted, E1- } \\
\text { E4 }\end{array}$ & 1.00 & 1.00 & 1.00 & 1.00 & 1.00 & 1.00 \\
\hline & \multirow{2}{*}{$\begin{array}{l}\text { Mid enlisted, E4-E6 } \\
\text { Senior enlisted, E5- } \\
\text { E9 }\end{array}$} & $\begin{array}{l}1.13(0.98- \\
1.30)\end{array}$ & $\begin{array}{l}1.07 \\
(0.96- \\
1.20)\end{array}$ & $\begin{array}{l}0.99 \\
(0.87- \\
1.11)\end{array}$ & $\begin{array}{l}1.02 \\
(0.92- \\
1.14)\end{array}$ & $\begin{array}{l}1.03 \\
(0.92- \\
1.16)\end{array}$ & $\begin{array}{l}1.13(0.85- \\
1.50)\end{array}$ \\
\hline & & $\begin{array}{l}1.38(1.12- \\
1.69)\end{array}$ & \multirow{2}{*}{$\begin{array}{l}1.26 \\
(1.08- \\
1.46)\end{array}$} & \multirow{2}{*}{$\begin{array}{l}0.90 \\
(0.77- \\
1.04)\end{array}$} & \multirow{2}{*}{$\begin{array}{l}0.99 \\
(0.86- \\
1.13)\end{array}$} & \multirow{2}{*}{$\begin{array}{l}0.87 \\
(0.75- \\
1.01)\end{array}$} & $\begin{array}{l}0.99(0.70- \\
1.38)\end{array}$ \\
\hline & Warrant officer & $\begin{array}{l}1.86(1.23- \\
2.79)\end{array}$ & & & & & $\begin{array}{l}0.93(0.56- \\
1.56)\end{array}$ \\
\hline & Junior officer, 01-03 & $\begin{array}{l}0.97(0.79- \\
1.20)\end{array}$ & $\begin{array}{l}1.62 \\
(1.25- \\
2.10)\end{array}$ & $\begin{array}{l}0.88 \\
(0.69- \\
1.11)\end{array}$ & $\begin{array}{l}0.87 \\
(0.70- \\
1.08)\end{array}$ & $\begin{array}{l}0.81 \\
(0.64- \\
1.03)\end{array}$ & $\begin{array}{l}0.84(0.58- \\
1.22)\end{array}$ \\
\hline & \multirow{2}{*}{ Senior officer, 04-09 } & \multirow[t]{2}{*}{$\begin{array}{l}1.36(1.06- \\
1.74)\end{array}$} & $\begin{array}{l}1.20 \\
(1.03- \\
1.40)\end{array}$ & $\begin{array}{l}1.06 \\
(0.90- \\
1.24)\end{array}$ & $\begin{array}{l}0.77 \\
(0.67- \\
0.89)\end{array}$ & $\begin{array}{l}0.56 \\
(0.48- \\
0.66)\end{array}$ & \multirow[t]{2}{*}{$\begin{array}{l}0.78(0.53- \\
1.15)\end{array}$} \\
\hline & & & $\begin{array}{l}1.27 \\
(1.07- \\
1.51)\end{array}$ & $\begin{array}{l}0.98 \\
(0.83- \\
1.16)\end{array}$ & $\begin{array}{l}0.94 \\
(0.80- \\
1.10)\end{array}$ & $\begin{array}{l}0.41 \\
(0.34- \\
0.49)\end{array}$ & \\
\hline \multirow{3}{*}{$\begin{array}{l}\text { Occupation } \\
\text { assignment group }\end{array}$} & Combat arms & 1.00 & 1.00 & 1.00 & 1.00 & 1.00 & 1.00 \\
\hline & \multirow{2}{*}{$\begin{array}{l}\text { Combat support } \\
\text { Combat service } \\
\text { support }\end{array}$} & \multirow{2}{*}{$\begin{array}{l}1.01(0.90- \\
1.13) \\
1.00(0.89- \\
1.12)\end{array}$} & $\begin{array}{l}0.92 \\
(0.85- \\
1.00)\end{array}$ & $\begin{array}{l}1.05 \\
(0.97- \\
1.13)\end{array}$ & $\begin{array}{l}1.04 \\
(0.97- \\
1.12)\end{array}$ & $\begin{array}{l}0.96 \\
(0.89- \\
1.04)\end{array}$ & $\begin{array}{l}1.09(0.92- \\
1.29)\end{array}$ \\
\hline & & & $\begin{array}{l}0.96 \\
(0.89- \\
1.04)\end{array}$ & $\begin{array}{l}1.00 \\
(0.93- \\
1.08)\end{array}$ & $\begin{array}{l}1.11 \\
(1.03- \\
1.19)\end{array}$ & $\begin{array}{l}0.92 \\
(0.85- \\
1.00)\end{array}$ & $\begin{array}{l}1.12(0.95- \\
1.33)\end{array}$ \\
\hline Service branch & Air Force & 1.00 & 1.00 & 1.00 & 1.00 & 1.00 & 1.00 \\
\hline & & $\begin{array}{l}1.02(0.92- \\
1.14)\end{array}$ & $\begin{array}{l}1.03 \\
(0.95-\end{array}$ & $\begin{array}{l}0.93 \\
(0.87-\end{array}$ & $\begin{array}{l}0.93 \\
(0.87-\end{array}$ & $\begin{array}{l}1.11 \\
(1.03-\end{array}$ & $\begin{array}{l}1.18(1.00- \\
1.40)\end{array}$ \\
\hline & Marıne Corps & $0.98(0.85-$ & & & & & $1.03(0.82-$ \\
\hline & Navy & $1.13)$ & $\begin{array}{l}1.09 \\
(0.98-\end{array}$ & $\begin{array}{l}0.90 \\
0.81-\end{array}$ & $\begin{array}{l}0.88 \\
0.80-\end{array}$ & $\begin{array}{l}1.26 \\
(1.15-\end{array}$ & $1.30)$ \\
\hline & & $\begin{array}{l}1.03(0.91- \\
1.16)\end{array}$ & $1.20)$ & $0.99)$ & $0.96)$ & $1.39)$ & $\begin{array}{l}1.18(0.99- \\
1.40)\end{array}$ \\
\hline & & & $\begin{array}{l}1.23 \\
(1.13- \\
1.33)\end{array}$ & $\begin{array}{l}1.10 \\
(1.02- \\
1.19)\end{array}$ & $\begin{array}{l}0.90 \\
(0.83- \\
0.97)\end{array}$ & $\begin{array}{l}0.90 \\
(0.82- \\
0.98)\end{array}$ & \\
\hline
\end{tabular}

Characteristics associated with higher overall caffeine use included female gender, older age, White race/ethnicity, higher BMI, less resistance training, current or former tobacco use, higher alcohol intake, and higher enlisted or officer rank. Higher coffee prevalence was associated with female gender, older age, higher formal education, White race/ethnicity, higher BMl, former or current tobacco use, higher alcohol intake, higher enlisted or officer rank, and service in the Navy (compared to the Air Force). Higher prevalence of tea was associated with female gender, older age, more formal education, Other race/ethnicity (compared to Whites), White race/ethnicity (compared to Hispanics), more aerobic exercise, less resistance training, current smoking, never using smokeless tobacco, higher alcohol intake, and service in the Navy (compared to the Air Force) or Air Force (compared to the Marine Corps). Higher soda prevalence was associated with male gender, less formal education, White race/ethnicity, higher BMI, less resistance training, current or former smoking, higher alcohol consumption, junior enlisted status (compared to junior officer status), and service in the Air Force (compared to all other services). Higher energy drink prevalence was associated with male gender, younger age, less formal education, White race/ethnicity, higher BMI, more resistance training, current or former tobacco use, higher alcohol consumption, lower enlisted rank (compared to officers), and service in the Army or Marine Corps (compared to the Air Force) or in the Air Force (compared to the Navy). Higher prevalence of caffeinated gums/medications use was independently associated with female gender, older age, White and Black race/ethnicity, higher BMI, higher alcohol intake, and service in the Army (compared to the Air Force). 


\section{Caffeine Consumption by Age and Sex}

Figure 1 presents daily caffeine consumption (mg/day) from all types of caffeinated products by age and sex. As age increased, there was a significant linear trend for increasing consumption of any caffeine and caffeine from coffee among both men and women $(p<0.01$, both sexes). In contrast, there was a significant linear trend for less consumption of energy drinks as age increased for both men and women ( $<0.01$, both sexes). While there was a significant linear trend of increased caffeine consumption from tea over age among men $(p=0.02)$, there was no such trend among women $(p=0.42)$. There were no significant linear trends over age for soda (men $p=0.07$, women $p=0.48$ ) or for gums/medications (men $p=0.13$, women $p=0.82$ ).

\section{Discussion}

This very large $(n=26,680)$, randomized, comprehensive assessment of SM caffeine consumption found $87 \%$ of SMs consumed caffeinated products, with an average estimated consumption of $243 \mathrm{mg} /$ day for consumers. Among SMs who regularly consumed caffeinated products, men consumed $29 \%$ more caffeine than women ( $251 \mathrm{vs} 195 \mathrm{mg} /$ day), but when caffeine consumption was adjusted for body weight, consumption was similar in men and women ( $2.93 \mathrm{vs} 2.85 \mathrm{mg} / \mathrm{day} / \mathrm{kg}$, respectively, $\mathrm{p}=0.12)$, as reported previously in the general population [1, 15]. Coffee was the beverage most frequently consumed (by $68 \%$ of the population), followed by soda (42\%), tea (29\%), energy drinks (29\%) and gums/candies/medications (4\%). Total caffeine intake from coffee was $69 \%$, energy drinks $17 \%$, tea $8 \%$, soda $6 \%$, and gums/medications $<1 \%$. Consuming any caffeinated product was independently associated with female gender, older age, White race/ethnicity, higher BMI, less resistance training, current or former tobacco use, higher alcohol intake, and higher enlisted or officer rank. Higher energy drink prevalence was associated with male gender, younger age, less formal education, White race/ethnicity, higher BMI, more resistance training, current or former tobacco use, higher alcohol consumption, lower enlisted rank (compared to officers), and service in the Army or Marine Corps (compared to the Air Force) or in the Air Force (compared to the Navy).

It is well documented that the civilian and military populations are generally aware of the effects of caffeine on human cognitive and physical performance. Surveys of SMs and college students found they consume caffeine-containing products for several reasons related to the performance benefits of caffeine $[19,28,29]$. Furthermore, SMs assigned to units in Afghanistan and likely to be engaged in combat consumed higher levels of caffeine than SMs at their home bases. Caffeine use by these SMs was higher among those reporting difficulty remaining awake during guard duty, poor sleeping conditions, and sleep disruptions during nighttime operations [18]. In addition, a survey of active duty Army aviators found they consumed more caffeine than their peers in non-aviation units, especially to enhance performance degraded due to insufficient sleep and very disruptive work schedules [19].

US Department of Defense laboratories and their international collaborators have conducted multiple studies designed to simulate military operations demonstrating the cognitive and physical benefits of caffeine consumption by military personnel [30-32]. The Department of Defense recognizes the ability of caffeine to enhance cognitive performance and provides it in rations, when necessary, with appropriate labeling to inform SMs of the presence and effects of caffeine [33].

\section{Caffeine Prevalence and Daily Consumption}

Previous studies have been conducted on caffeine prevalence and daily consumption among Air Force [22], Army [20], and Navy/Marine Corps [21] personnel. All of these studies [20-22] used a slightly different questionnaire but the same definitions for caffeine sources. The Air Force [22] and Army [20] studies used a convenience sampling technique involving volunteers in face-to-face administrations at installations across the US and overseas, and the Navy and Marine Corps study [21] identified a random sample and asked for volunteers by postal letter and e-mail. The present study was quite similar to the Navy/Marine Corps study [21] in that a random sample of SMs were studied, but the questionnaire differed from that of previous studies [20-22]. Those studies listed not only generic sources of caffeine (e.g., coffee, tea, soft drinks), as in the present study, but specific products (e.g., Dr. Pepper soda, Monster energy drink, No Doz Gum) as well. Given these differences in study design, Table 4 compares caffeine use prevalence and daily consumption among the military services in the current and past studies. Prevalence estimates for any caffeinated product ( $\geq$ $1 /$ week) were similar across all studies. With regard to individual caffeinated products, the previous Army and Navy/Marine Corps studies $[20,21]$ found the highest consumption prevalence for coffee, but Air Force personnel were unique in that cola was the most ingested product, with coffee ranking second [22]. The current study found that in all services, coffee was the product consumed most often. Daily caffeine consumption estimates were similar for Air Force personnel in the current and past [22] investigations, but

Page $16 / 25$ 
estimates for Army, Navy, and Marine Corps personnel were 38\% lower, 21\% higher, and 16\% higher, respectively [20, 21]. Differences in estimation of caffeine consumption from individual products in past studies [20-22] versus estimates from generic types (coffee, tea, soda) in the current study likely accounted for these differences. Most past studies [20, 21] and the current one agree in that SMs in all services consumed the most total caffeine ( $\mathrm{mg} /$ day) from coffee, with energy drinks ranking second.

Table 4

Comparison of studies on prevalence and amount of caffeine consumption in military services $^{a}$

\begin{tabular}{|c|c|c|c|c|c|}
\hline Measure & Study & Air Force & Army & Marine Corps & Navy \\
\hline \multirow[t]{2}{*}{ Prevalence $(\%)^{b}$} & Previous $^{c}$ & 84 & 82 & 86 & 88 \\
\hline & Current & 86 & 87 & 85 & 89 \\
\hline \multirow[t]{2}{*}{ Daily Consumption (mg/day) } & Previous $^{c}$ & 212 & 347 & 232 & 217 \\
\hline & Current & 215 & 252 & 270 & 264 \\
\hline \multicolumn{6}{|l|}{${ }^{\mathrm{a} C}$ affeine consumers only } \\
\hline \multicolumn{6}{|c|}{${ }^{\mathrm{b}}$ Caffeine prevalence is defined as use $\geq 1$ time/week. } \\
\hline
\end{tabular}

Several population-based estimates of caffeine consumption in adult Americans based on very large population samples using stateof-the-art dietary intake procedures are available. NHANES caffeine intake $[1,13,15]$ was calculated based on 24-hour dietary recalls in 2001-2012. Estimated caffeine use prevalence in adults (>19 years) was $89 \%$ for men and $89 \%$ for women [1]. Caffeine consumption estimates for consumers of caffeine varied from 189 to $211 \mathrm{mg} /$ day for men and 149 to $161 \mathrm{mg} /$ day for women [1, 13, 15]. The Kantar Worldwide Beverage Consumption Panel obtained data on US consumers from an online, 7-day beverage consumption record and found $\sim 90 \%$ of individuals $\geq 18$ years of age consumed caffeinated beverages, with average caffeine consumption equal to about $200 \mathrm{mg} /$ day among caffeine consumers (males and females were not separated) [2]. Prevalence values in these population-based studies were similar to the $87 \%$ observed in SMs ( $\geq 1$ week), while the average consumption in SMs of 251 and $195 \mathrm{mg} /$ day for males and females, respectively, was somewhat higher than in the civilian population.

At least three other surveys [20-22] of the individual branches of service have observed caffeine-intake levels similar to those reported here and higher than those in the civilian population. The extensive and unique demands of military service may be a factor that explains the difference in caffeine intake in military versus civilian personnel. Differences in the methods and the demographic characteristics of the samples used in civilian studies and the current investigation must also be considered when interpreting these differences. For example, active duty SMs are younger, fully employed, and sleep somewhat less than the general population [16].

The estimated average daily caffeine consumption of military personnel who are regular caffeine consumers was well below the levels that are widely recognized as safe: $400 \mathrm{mg} /$ day for men and $300 \mathrm{mg} /$ day for women of reproductive age [5-7]. Nonetheless, the present study found that caffeine consumption of $~ 15 \%$ of men and $8 \%$ of women exceeded $400 \mathrm{mg} / \mathrm{day}$, and that of $15 \%$ of women exceeded $300 \mathrm{mg} /$ day. These proportions are very similar to those found in past military studies [21, 22]. Some individuals may be able to consume higher amounts of caffeine without adverse effects, although this cannot be determined from the current data. A genetic polymorphism allows some individuals to metabolize ( $\mathrm{N}^{3}$-demethylation) caffeine in the liver more rapidly than others, and another polymorphism may be associated with higher caffeine tolerance and consumption [34-36].

Energy drink use prevalence ( $\geq 1$ time/week) was $29 \%$ in the present study and varied from $21-39 \%$ in the military studies [20-22, 37-39]. Various studies of energy drink consumption among US college students found that $39 \%$ reported consuming in the past week [40], 36\% within the past 2 weeks [41] and 36\% within the past year [28]. Data from several NHANES cycles indicated that prevalence of daily consumption of energy drinks among adults has increased from 2003 to 2016 [10]. With regard to caffeine consumption, the current study found that $17 \%$ of the total caffeine was consumed from energy drinks. Data from NHANES suggested only $1-2 \%$ of total caffeine consumed by Americans was from energy drinks [1, 13], but a study of a convenience sample of geographically dispersed college students in the US found $22 \%$ of their total caffeine consumption was from energy drinks [28]. In summary, the prevalence of energy drink consumption by SMs, and the proportion of total caffeine consumption from energy drinks

Page $17 / 25$ 
by SMs, are similar to those of college students- despite the generally older age of SMs-and much higher than those of the general US population.

\section{Characteristics Associated with Caffeine Consumption}

In the univariate analysis, there was little difference in the prevalence of consumption of caffeinated products and coffee by men and women. In the multivariate analysis, however, women had greater odds of consumption than men. This was primarily due to the influence of alcohol consumption in the statistical models, although smoking and smokeless tobacco also had minor effects. Caffeine consumption increased as alcohol intake increased, or if individuals were tobacco users; men were more likely to be higher alcohol consumers or tobacco users. Alcohol or tobacco use accounted for a larger proportion of the odds ratio for the effect of sex on caffeine consumption in men than in women. Because of this, the odds of consuming caffeine became lower in men than in women. Dividing the higher odds of caffeine intake in women by the lower odds of caffeine intake in men resulted in the larger odds ratio for women for any caffeinated product and coffee. If alcohol consumption, smoking, and smokeless tobacco use were not included in models 1 and 2 (Table 3 ) the odds ratios (95\% confidence intervals) for women (compared to men) were 1.02 (0.92-1.14) and 1.02 (0.94-1.11), respectively.

In agreement with the current study, others $[1,12,13,20,21]$ have reported that men consumed more caffeine than women. Nonetheless, this study and others $[12,21,22]$ found that when caffeine consumption was determined on a per kg body weight basis, men and women consumed similar amounts. Although coffee was the major source of caffeine for both men and women, female SMs consumed more caffeine from tea while male SMs consumed more caffeine from soda and energy drinks. Acute caffeine consumption modestly affects moods such as vigor and fatigue as well as hemodynamic measures (e.g. blood pressure, cardiac output) in men and women [42-44], although cardiovascular effects are more likely to be observed at higher doses. Both men and women report consuming caffeinated products to provide behavioral benefits such as increased alertness [19, 28, 29].

Investigations involving representative civilian $[1,2,12,13,27]$ and military $[21,22]$ samples reported that overall use and/or amount of caffeine consumption increased with age, although prevalence and/or caffeine amounts decline at the highest age groups (generally $>65$ years) $[1,2,12,13,27]$. Also in general agreement with past military studies [20-22], the current study found that coffee consumption accounted for most of the caffeine ingested in all age groups, but younger ( $<40$ years) individuals consumed over twice as much caffeine from energy drinks as older ( $\geq 40$ years) individuals (46 vs $22 \mathrm{mg} / \mathrm{day}, \mathrm{p}<0.01$ ) and were almost twice as likely to consume energy drinks (33 vs 17\%, p<0.01). Energy drinks were introduced into the American market in 1997 [45], and their advertising was targeted to teenagers and individuals in 18- to 34-year-olds [46]. This advertising may have influenced energy drink consumption in the younger age groups in the current study.

Other civilian $[13,15]$ and military [20-22] studies have reported that compared to Whites, Blacks have a lower prevalence of caffeine use and a lower total caffeine consumption, accounted for largely by less coffee consumption [20-22, 47]. There are race/ethnic differences in dietary intake $[48,49]$, and some of these differences appear to be partly accounted for by educational level and income $[49,50]$. In the current study, differences between Black and White SMs in caffeine and coffee prevalence remained after controlling for formal educational level, rank (a surrogate for income), and other factors, in agreement with past military studies [21, 22]. The reasons for the race/ethnic differences are likely complex and may be different in the military compared to the general population.

In agreement with other investigations [20,21,28], the current study found no systematic association between weekly aerobic exercise duration and caffeine use prevalence. One study of Air Force personnel [22] found prevalence decreased with increased aerobic activity duration; in the current study, when Air Force personnel were considered separately, this relationship was not duplicated (data not shown). For resistance training, both univariate and multivariable analysis showed the lowest caffeine use prevalence in the group exercising the most with little difference among the other groups, in general agreement with most other military studies [21, 22]. One study which separated Army personnel into those who performed weight training and those who did not found that trainers had higher overall use prevalence [20], also in agreement with the current study. Previous military studies have shown that dietary supplement use was strongly associated with increasing resistance training duration [21,23]. Many dietary supplements contain caffeine, and the caffeine content of some of these can be very high [51]. Accurately determining the caffeine content of dietary supplements is difficult because manufactures are not required to list the amount of caffeine on their supplement facts labels, amounts are usually not available on company websites, and if the ingredients are proprietary, the manufacturer is not required to list caffeine at all [51]. It is possible that SMs involved in large amounts of resistance training consumed less caffeine from beverages to avoid adverse effects resulting from high dosages of caffeine in their dietary supplements. Overall, the current data and previous investigations suggest 
little relationship between aerobic exercise duration and caffeine use prevalence, but for resistance training there appears to be a bimodal relationship such that those performing the least or the most training have lower use prevalence than those performing moderate amounts of training.

Current or former tobacco use (smoking or smokeless tobacco) was associated with a higher use and higher intake of caffeine, especially for coffee and energy drinks, in both univariate and multivariable analyses. Although associations with smokeless tobacco have not been previously investigated, associations between caffeine prevalence and smoking have repeatedly been reported in both military $[20,22]$ and civilian populations $[14,15,52-58]$. Smoking accelerates caffeine metabolism and reduces its half-life [59, 60] suggesting that smokers consume more caffeine to achieve stimulatory effects. In addition, both caffeine and smoking increase dopaminergic activity in different brain regions, and the two substances may be used concurrently to potentiate stimulation [61]. Studies of monozygotic and dizygotic twins suggest that both genetic and environmental factors may be involved in the relationship between tobacco use and caffeine [62-65] with genetic factors becoming more important as individuals age [64]. There is also specific genetic evidence for a casual effect of smoking on caffeine intake. Specific single nucleotide polymorphisms (SNPs rs16969968/rs1051730) on the nicotinic receptor subunit gene (CHRNA5) increase daily cigarette consumption among smokers. As the number of these SNPs increases, so does coffee consumption [58].

Another lifestyle factor strongly associated with caffeine prevalence and consumption was alcohol intake. In both univariate and multivariable analyses, use prevalence of caffeinated products of all types increased in a dose-response manner as alcohol consumption increased. The amount of caffeine consumed from coffee and energy drinks increased as alcohol intake increased. Similar relationships have been found in other studies for coffee $[14,21,56,66]$, energy drinks [21, 67-69], and overall caffeine use $[15,21,69]$. Studies of monozygotic and dizygotic twins suggested that there was a common genetic factor underlying this association, but environmental influences still seemed to contribute to the variance in caffeine consumption $[63,64,70]$. A recent study based on variations in SNPs support that the genes underlying the use of both coffee and alcohol were heritable [71]; however, two-sample Mendelian randomization suggested there was no causal association between coffee consumption and alcohol consumption [71, 72]. Psychosocial factors may play a role in this association since studies have consistently shown that higher levels of sensation-seeking behaviors are associated with both higher caffeine and alcohol use [73-75].

In the current study, SMs who reported less daily sleep consumed more caffeine for all sources, in agreement with past military [21, 22, 76] and civilian [77] studies. Military personnel sleep less than civilian populations [16, 17] and averaged 6.3 hours in current study, less than the recommended $\geq 7$ hours/night [78]. Military training and operations can occur at any time of the day, can extend continuously for many days, and can involve substantial loss of sleep. Caffeine can increase alertness due to its ability to block central adenosine receptors [79]; when ingested in sufficient dosages, it can reduce sleep duration [80], and it improves cognitive performance, especially vigilance [81-83].

\section{Strengths and Limitations}

A major strength of this study was recruitment of a very large, stratified random sample of SMs who answered a standard set of questions on their consumption of specific caffeinated products. With a few exceptions, the data largely confirm results of past investigations of caffeine prevalence and consumption involving smaller studies of separate military services, using convenience samples [20-22]. Nonetheless, there are several limitations to the current analyses, most of which relate to difficulty in estimating daily caffeine consumption. First, all data were self-reported, and the usual shortcomings associated with this method, including recall bias, social desirability, errors in self-observation, and inadequate recall, apply [84, 85]. These biases could account for errors in reporting serving sizes and how many times per week SMs used caffeinated products and, as a consequence, errors in estimating caffeine consumption. Second, caffeine data for this study were obtained from beverages and gums/medications; we purposely did not assess caffeine intake from food sources as beverages account for $98 \%$ of caffeine consumption [1]. Third, caffeine contents of products were estimated based on standardized values of each type of caffeinated product. Specific products can differ in caffeine content [26, 86-88]. Finally, a large number of statistical tests examining relationships between caffeine prevalence and consumption and the demographic, lifestyle, and military characteristics were conducted, thus increasing the probability of Type 1 errors.

\section{Conclusions}

Among all military personnel surveyed, $87 \%$ reported consuming caffeinated products $\geq 1$ time/week, with male and female consumers ingesting (mean \pm standard error) $251 \pm 2$ and $195 \pm 3 \mathrm{mg} /$ day, respectively. The prevalence of caffeine consumption by 
military personnel was similar to that reported in NHANES data, but total caffeine consumption was higher. Compared to civilians, SMs may consume more caffeine to enhance their cognitive and physical performance due to the intense occupational demands of their profession. The most commonly consumed caffeinated products (\% users) were coffee (68\%), soda (42\%), tea ( $29 \%)$, and energy drinks (29\%). Coffee, tea, soda, energy drinks, and gums/medications accounted for $69 \%, 8 \%, 6 \%, 17 \%$, and $>1 \%$ of total caffeine consumption, respectively. The prevalence of energy drinks consumption and amount of caffeine ingested from energy drinks was about twice as high among those $<40$ years of age compared to those $\geq 40$ years of age. Characteristics associated with caffeine use in SMs were generally similar to those observed in investigations of civilians.

\section{Abbreviations}

ANOVA

Analysis of variance

BMI

body mass index

NHANES

National Health and Nutrition Examination Survey

SM

service member

SE

standard error

US

United States

\section{Declarations}

Ethics Approval and Consent to Participate: The Naval Health Research Center's Institutional Review Board approved the investigation (protocol number NHRC.2016.0025). SMs consented to participate by signing an informed consent document. Investigators adhered to policies and procedures for protection of human subjects as prescribed by Department of Defense Instruction 3216.01, and the research was conducted in adherence with provisions of 32 Code of Federal Regulations Part 219.

Consent for Publication: NA

Availability of Data and Materials: The datasets generated and/or analyzed during the current study are not publicly available due to US government restrictions, but are available from the corresponding author on reasonable request.

Competing Interests: The authors declare that they have no competing interests.

Funding: This work was funded by Department of Defense Center Alliance for Nutrition and Dietary Supplement Research of the Defense Medical Research and Development Program, the US Army Medical Research and Development Command (USAMRDC). The Bureau of Medicine and Surgery also supported this work under Work Unit No. N1335.

Authors' Contributions: JJK designed the research, analyzed data, wrote paper, and had responsibility for final content; TWD designed research, conducted research, provided essential material, and had responsibility for final content; RAS analyzed data and had responsibility for final content; EKF designed research and had responsibility for final content; HRL designed research and had responsibility for final content. All authors have read, edited, and approved the final manuscript.

Acknowledgements: Thanks to Dr. Kathryn Taylor for assistance in interpreting the data, to Ms Maureen Humphrey-Shelton for assistance in obtaining references, and to Ms Lauren Thompson for editorial comments.

Authors Information: The opinions contained herein are the private views of the authors and are not to be construed as official or as reflecting the views of the Army or the Department of Defense. Citations of commercial organizations and trade names in this report do not constitute an official Department of the Army endorsement or approval of the products or services of these organizations.

Approved for public release; distribution is unlimited.

Page 20/25 
The authors are military service members or employees of the US Government. This work was prepared as part of their official duties. Title 17, U.S.C. $\S 105$ provides that copyright protection under this title is not available for any work of the US Government. Title 17, U.S.C. §101 defines a U.S. Government work as work prepared by a military service member or employee of the US Government as part of that person's official duties. Part of this work was supported by Defense Health Program under work unit no. N1335. The views expressed in this article are those of the authors and do not necessarily reflect the official policy or position of the Department of the Army, Navy, Department of Defense, nor the U.S. Government. The study protocol was approved by the Naval Health Research Center Institutional Review Board in compliance with all applicable Federal regulations governing the protection of human subjects. Research data were derived from an approved Naval Health Research Center Institutional Review Board protocol, number NHRC.2016.0025.

\section{References}

1. Fulgoni VL, Keast DR, Lieberman HR: Trends in intake and sources of caffeine in the diet of US adults: 2001-2010. American Journal of Clinical Nutrition 2015, 101:1081-1087.

2. Mitchell DC, Knight CA, Hokenberry J, Teplansky R, Hartman TJ: Beverage caffeine intakes in the U.S. Food and Chemical Toxicology 2014, 63:136-142.

3. Cappelletti S, Piacentino D, Fineschi V, Frati P, Cipolloni L, Aromatario M: Caffeine-realated deaths; manner of deaths and categories at risk. Nutrients 2018, 10:611.

4. Koert RRv, Bauer PR, Schuitema I, Sander JW, Visser GH: Caffeineand seizures: a systematic review and quantitative analysis. Epilepsy and Behavior 2018, 80:37-47.

5. Scientific report of the 2015 Dietary Guidelines Advisory Committee [https://health.gov/dietaryguidelines/2015-scientificreport/pdfs/scientific-report-of-the-2015-dietary-guidelines-advisory-committee.pdf] Accessed 7 October 2016.

6. Nawrot P, Jordan S, Eastwood J, Rotstein J, Hugenholtz A, Feeley M: Effects of caffeine on human health. Food Additives \& Contaminants 2003, 20(1):1-30.

7. Wikoff D, Welsh BT, Henderson R, Bororby GP, Britt J, Myers E, Goldberger J, Lieberman HR, O'Brien C, J. Peck MT, et al: Systematic review of the potential adverse effects of caffeine consumption in healthy adults, pregnant women, adolescents, and children. Food and Chemical Toxicology 2017, 109:585-648.

8. Grosso G, Godos J, Galvano F, Giovannucci EL: Coffee, caffeine, and health: an umbrella review. Annual Review of Nutrition 2017, 37:131-156.

9. Kim Y, Je Y, Giovannucci E: Coffee consumption and all-cause and cause specific mortality: a meta-analysis by potential confounders. European Journal of Epidemiology 2019, 34(8):731-752.

10. Vercammen KA, Koma JW, Bleich SN: trends in energy drink consumption among U.S. adolescents and adults, 2003-2016. American Journal of Preventive Medicine 2019, 56(6):827-833.

11. Branum AM, Rossen LM, Schoendorf KC: Trends in caffeine intake among US children and adolescents. Pediatrics 2014, 133(3):386-393.

12. Frary CD, Johnson RK, Wang MQ: Food sources and intakes of caffeine in the diets of persons in the United States. Journal of the American Dietetic Association 2005, 105:110-113.

13. Drewnowski A, Rehm CD: Sources of caffeine in diets of US children and adults: trends by beverage type and purchase location. Nutrients 2016, 8:154.

14. Loftfield E, Freedman ND, Dodd KW, Vogtmann E, Xiao Q, Sinha R, Graubard BI: Coffee drinking is widespread in the United States, but usual intake varies by key demographic and lifestyle factors. Journal of Nutrition 2016, 146:1762-1768.

15. Lieberman HR, Agarwal S, Fulgoni VL: Daily pattern of caffeine intake with multiple sociodemographic and lifestyle factors in US adults based on NHANES 2007-2012 surveys. Journal of the Academy of Nutrition and Dietetics 2019, 119(1):106-114.

16. Good CH, Brager AJ, Capaldi VF, Mysliwiec V: Sleep in the United States military. Neuropsychopharmacology Reviews 2019, 45:176-191.

17. Krueger PM, Friedman EM: Sleep duration in the United states: a cross sectional population-based study. American Journal of Epidemiology 2009, 169(9):1052-1063.

18. McLellan TM, Riviere LA, Williams KW, McGurk D, Lieberman HR: Caffeine and energy drink use by combat arms soldiers in Afghanistan as a countermeasure for sleep loss and high operational demands. Nutritional Neuroscience 2019, 22(11):768-777.

Page 21/25 
19. Bukhari AS, Caldwell JA, DiChiara AJ, Merrill EM, Wright AO, Cole RE, Hatch-McChesney A, McGraw SM, Lieberman HR: Caffeine, energy beverage consumption, fitness, and sleep in U.S. Army aviation personnel. Aerospace Medicine and Human Performance 2020, 91(8):641-650.

20. Lieberman HR, Stavinoha T, McGraw S, White A, Hadden L, Marriott BP: Caffeine use among active duty US Army soldiers. Journal of the Academy of Nutrition and Dietetics 2012, 112(6):902-912.

21. Knapik JJ, Trone DW, McGraw S, Steelman RA, Austin KG, Lieberman HR: Caffeine use among active duty Navy and Marine Corps personnel Nutrients 2016, 6:620.

22. Knapik JJ, Austin KG, McGraw SM, Leahy GD, Lieberman HR: Caffeine consumption among active duty United States Air Force personnel. Food and Chemical Toxicology 2017, 105:377-386.

23. Knapik JJ, Trone DW, Steelman RA, Farina EK, Lieberman HR: Prevalence and factors associated with diatary supplement use in a stratified random sample of United States military personnel: the US Military Dietary Supplement Use Study. Journal of Nutrition 2021, 151(11):3495-3506.

24. Edwards P, Cooper R, Roberts I, Frost C: Meta-analysis of randomized trials of monetary incentives and response to mailed questionnaires. Journal of Epidemiology and Community Health 2005, 59:987-999.

25. Church $\mathrm{AH}$ : Estimating the effect of incentives on mail survey response rates: a meta-analysis. Public Opinion Quarterly 1993, 57:62-79.

26. Caffeine Content of Drinks [http://www.caffeineinformer.com/the-caffeine-database] Accessed 22 June 2021.

27. Knight CA, Knight I, Mitchell DC, Zepp JE: Beverage caffeine intake in the US consumers and subpopulations of interest: estimates from the Share of Intake Panel survey. Food and Chemical Toxicology 2004, 42(12):1923-1930.

28. Mahoney CR, Giles GE, Marriott BP, Judelson DA, Glickman EL, Geiselman PJ, Lieberman HR: Intake of caffeine from all sources and reasons for use by college students. Clinical Nutrition 2019, 38(2):668-675.

29. Mcllvain GE, Noland MP, Bickel R: Caffeine consumption patterns and beliefs of college freshman. American Journal of Health Education 2011, 42(4):2011.

30. Kaminori GH, McLellan TM, Tate CM, Voss DM, Niro P, Lieberman HR: Caffeine improves reaction time, vigilance and logical reasoning during extended periods with restricted oppertunities for sleep. Psychopharmacology 2015, 232:2013-2042.

31. Lieberman HR, Tharion WJ, Shukitt-Hale B, Speckman KL, Tulley R: Effects of caffeine, sleep loss, and stress on cognitive performance and mood during US Navy SEAL training. Psychopharmacology 2002, 164:250-261.

32. McLellan TM, Bell DG, Lieberman HR, Kamimori GH: The impact of caffeine on cognitive and physical performance and marksmanship during sustained operations. Canadian Military Journal 2004, 4(4):47-54.

33. Chapter 11: Mission Nutrition for Combat Effectiveness [https:// Accessed 5 March 2021.

34. Cornelis MC, Al-Sohemy A, Campos $\mathrm{H}$ : Genetic polymorphism of the adenosine A2A receptor is associated with habitual caffeine consumption. American Journal of Clinical Nutrition 2007, 86:240-244.

35. Sachse C, Brockmoller J, Bauer S, Roots I: Functional significance of a C to A polymorphism in intron 1 of the cytochrome P450 CYP1A2gene tested with caffeine. British Journal of Clinical Pharmacology 1999, 47:445-449.

36. Huang ZL, Qu WM, Eguchi N, Chen JF, Schwarzschild MA, Fredholm BB, Urade Y, Hayaishi O: Adenosine $A_{2 A}$ but not $A_{1}$ receptors mediate the arousal effect of caffeine. Nature Neuroscience 2005, 8(7):858-859.

37. Schmidt RM, Mclntire LK, Caldwell JA, Hallman C: Prevalence of energy-drink and supplement usage in a sample of Air Force personnel. Wright-Patterson Air Force Base: Air Force Research Laboratory; 2008.

38. Stephens MB, Attipoe AS, Jones D, Ledford CJW, Deuster PA: Energy drink and energy shot use in the military. Nutrition Reviews 2014, 72 (Suppl 1):72-77.

39. Jacobson IG, Horton JL, Smith B, Wells TS, Boyko EJ, H.R L, Ryan MAK, Smith TC: Bodybuilding, energy, and weight loss supplements are associated with deployment and physical activity in US military personnel. Annals of Epidemiology 2012, 22(5):318-330.

40. Skewes MC, Decou CR, Gonzalez VM: Energy drink use, problem drinking and drinking motives in a diverse sample of Alaska college students. International Journal of Circumpolar Health 2013, 72:21204.

41. Marczinski CA: Alcohol mixed with energy drinks: consumption patterns and motivations for use in U.S. college students. International Journal of Environmental Research and Public Health 2011, 8:3232-3245.

Page 22/25 
42. Hartley TR, Lovallo WR, Whitsett TL: Cardiovascular effects of caffeine in men and women. American Journal of Cardiology 2004, 93:1022-1026.

43. Farag NH, Whitsett TL, McKey BS, Wilson MF, Vincent AS, Everson-Rose SAl, Lovallo WR: Caffeine and blood pressure response: sex, age and hormonal status. Journal of Women's Health 2010, 19(6):1171-1176.

44. Amendola CA, Gabrieli JDE, Lieberman HR: Caffeine's effect on performance and mood are independent of age and gender. Nutritional Neuroscience 1998, 1:269-280.

45. Heckman MA, Sherry K, GonzalezDeMejia E: Energy drinks: assessment of their market size, consumer demographics, ingredient profile, functionality, and regulations in the United States. Comprehensive Reviews in Food Science and Food Safety 2010, 9:303317.

46. Lal GG: Getting specific with functional beverages. Food Technology 2007, 61(12):25-31.

47. Rehm CD, Ratliff JC, Riedt CS, Drewnowski A: Coffee consumption among adults in the United States by demographic variables and purchase location: analysis of NHANES 2011-2016 data. Nutrients 2020, 12:2463.

48. Hiza HAB, Casavale KO, Guenther PM, Davis CA: Diet quality of Americans differ by age, sex, race/ethnicity, income and educational level. Journal of the Academy of Nutrition and Dietetics 2013, 113(2):297-306.

49. Wang $Y$, Chen $X$ : How much of racial/ethnic disparities in dietary intake, exercise, and weight status can be explained by nutritionand health-related psychosocial factors and socioeconomic status among US adults. Journal of the American Dietetic Association 2011, 111:1904-1991.

50. Bahr PR: Race and nutrition: an investigation of Black-White differences in health-related nutrition behaviors. Sociology of Health and IIIness 2007, 29(6):831-856.

51. Andrews KW, Schweitzer A, Zhao C, Holden JM, Roseland JM, Brandt M, Dwyer JT, Picciano MF, Saldanha LG, Fisher KD, et al: The caffeine contents of dietary supplements commonly purchased in the US: analysis of 53 products with caffeine-containing ingredients. Analytical and Bioanalytical Chemistry 2007, 389(1):231-239.

52. Hewlett P, Smith A: Correlates of daily caffeine consumption. Appetite 2006, 46:97-99.

53. Swanson JA, Lee JW, Hopp JW: Caffeine and nicotine: a review of their joint use and possible interactive effects in tobacco withdrawal. Addictive Behaviors 1994, 19(3):229-256.

54. Treur JL, Taylor AE, Ware JJ, McMahon G, Hottenga JJ, Baselmans BML, Willemsen G, Boomsma DI, Munafo MR, Vink JM: Association between smoking and caffeine consumption in two European cohorts. Addiction 2016, 111:1059-1068.

55. Friis K, Lyng Jl, Lasgaard M, Larsen FB: Energy drink consumption and the relation to socio-demographic factors and health behaviors among young adults in Denmark. A population-based study. European Journal of Public Health 2014, 24(5):840-844.

56. Istvan J, Matarazzo JD: Tobacco, alcohol, and caffeine use: a review of their interrelationships. Psychological Bulletin 1984, 95(2):301-326.

57. Elhadad MA, Karavasiloglou N, Wulaningsih W, Tsilidis KT, Tzoulaki I, Patel CJ, Rohrmann S: Metabolites, nutrients and lifestyle factors ib relation to coffee consumption: an environment-wideassociation study. Nutrients 2020, 12:1470.

58. Bjorngaard JH, Nordestgaard AT, Taylor AE, Treur JL, Gabrielsen ME, Munafo MR, Nordestgaard BG, Asvold BO, Romundstad P, Smith GD: Heavy smoking increase coffee consumption: finding from a Mendelian ramdomization analysis. International Journal of Epidemiology 2017, 46(6):1958-1967.

59. Brown CR, Jacob P, Wilson M, Benowitz NL: Changes in rate and pattern of caffeine metabolism after cigarette abstinence. Clinical Phamacology and Theraputics 1988, 43(5):488-491.

60. Parsons WD, Nelms AH: Effects of smoking on caffeine clearence. Clinical Pharmacology and Theraupetics 1978, 24(1):40-45.

61. Tanda G, Goldberg SR: Alternations of the behavioral effects of nicotine by chronic caffeine exposure. Pharmacology Biochemistry and Behavior 2000, 66(1):47-64.

62. Swan GE, Carmelli D, Cardon LR: Heavy consumption of cigarettes, alcohol and coffee in male twins. Journal of Studies on Alcohol 1997, 58(2):182-190.

63. Hettema JM, Corey LA, Kendler KS: A multivariate genetic analysis of the use of tobacco, alcohol and caffeine in a population based sample of male and female twins. Drug and Alcohol Dependence 1999, 57:69-78.

64. Kendler KS, Schmitt E, Aggen SH, Prescott CA: Genetic and environmental influences on alcohol, caffeine, cannabis and nicotine use from early adolescence to middle adulthood. Archives of General Psychiatry 2008, 65(6):674-682. 
65. Treur JL, Taylor AE, Ware JJ, Nivard MG, Neale MC, McMahon G, Hottenga JJ, Baselmans BML, Boomsma DI, Munafo MR, Vink JM: Smoking and caffeine consumption: a genetic analysis of their association. Addiction Biology 2016, 22(4):1090-1102.

66. Kozlowski LT, Henningfield JE, Keenan RM, Lei H, Jelinek LC, Leigh G, Pope MA, Haertzen CA: Patterns of alcohol, cigarette, and caffeine and other drug use in two drug abusing populations. Journal of Substance Abuse Treatment 1993, 10:171-179.

67. Miller KE: Energy drinks, race, and problem behaviors among college students. Journal of Adolescent Health 2008, $43: 490-497$.

68. Velazquez CE, Poulos NS, Latimer LA, Pasch KE: Associations between energy drink consumption and alcohol use behaviors among college students. Drug and Alcohol Dependence 2012, 123:167-172.

69. Dillon P, Kelpin S, Kendler K, Thacker L, Dick D, Svikis D: Gender differences in any-source caffeine and energy drink use and associated adverse health behaviors. Journal of Caffeine and Adenosine Research 2019, 9(1):12-19.

70. Swan GE, Carmelli D, Cardon LR: The consumption of tobacco, alcohol, and coffee in Caucasian male twins: a multivariate genetic analysis. Journal of Substance Abuse 1996, 8(1):19-31.

71. Chang LH, Ong JS, An J, K JHV, Vink JM, Pasman J, Liu M, MacGregor S, Cornelis MC, Martin NG, Derks EM: Investigating the genetic and causal relationship between initiation or use of alcohol, caffeine, cannabis and nicotine. Drug and Alcohol Dependence 2020, 210:107966.

72. Verweij KJH, Treur JL, Vink JM: Investigating causal associations between use of nicotine, alcohol, caffeine and cannabis: a twosample bidirectional Mendelian randomization study. Addiction 2018, 113:1333-1338.

73. Mahoney CR, Giles GE, Williams CS, Brunye TT, Taylor HA, Kanarek RB, Marriott BP, Lieberman HR: Relationships between use of dietary supplements, caffeine and sensation seeking among college students. Journal of the American College of Health 2019 , 67(7):688-697.

74. Arria A, Caldeira KM, Kasperski SJ, O'Grady KE, Vincent KB, Griffiths RR: Increased alcohol consumption, nonmedical prescription drug use, and illicit drug use are associated with energy drink consumption among college students. Journal of Addiction Medicine 2010, 4(2):74-80.

75. Jones HA, Lejuez CW: Personality correlates of caffeind dependence: the role of sensation seeking, implusivity, and risk taking. Experimental and Clinical Psychopharmacology 2005, 13(3):259-266.

76. Toblin RL, Clarke-Walper K, Kok BC, Sipos ML, Thomas JL: Energy drink consumption and its association with sleep problems among U.S. service members on a combat deployment-Afghanistan, 2010 MMWR 2012, 61(44):895-898.

77. Kant AK, Graubard BI: Association between self-reported sleep duration with eating behaviors of American adults: NHANES 20052010. American Journal of Clinical Nutrition 2014, 100:938-947.

78. Watson NF, Badr S, Belenky G, Bliwise DL, Buxton OM, Buysse D, Dinges DF, Gangwisch J, Grandner MA, Kushida C, et al: Recommended amount of sleep for healthy aduts: a joint consensus statement of the American Academy of Sleeep Medicine and Sleep Research Society. Journal of Clinical Sleep Medicine 2015, 11(6):591-592.

79. Ribeiro JA, Sebastiao AM: Caffeine and adenosine. Journal of Alzheimer's Disease 2010, 20:S3-S15.

80. Clark I, Landolt HP: Coffee, caffeine and sleep: a systematic review of of epidemiological studies and randomized controlled trials Sleep Medicine Review 2017, 31:70-78.

81. Kamimori GH, McLellan TM, Tate CM, Voss DM, Niro P, Liberman HR: Caffeine improves reaction time, vigilance and logical reasoning during extended periods with restricted oppertunities for sleep. Psychopharmacology 2015, 232(12):2031-2042.

82. Lieberman HR, Tharion WJ, Shukitt-Hale B, Speckman KL, Tulley R: Effects of caffeine, sleep loss, and stress on cognitive performance and mood during U.S. Navy SEAL training. Psychopharmacology 2002, 164:250-261.

83. Mclellan TM, Caldwell JA, Lieberman HR: A review of caffeine's effect on cognitive, physical, and occupational performance. Neuroscience and Biobehavioral Reviews 2016, 71:294-312.

84. Podsakoff PM, MacKenzie SB, Lee JY, Podsakoff NP: Common method biases in behavioral research: a critical review of the literature and recommended remedies. Journal of Applied Psychology 2003, 88(5):879-903.

85. Furnham A: Response bias, social desirability and dissimulation. Personality and Individual Differences 1985, 7(3):385-400.

86. Hecimovic I, Belscak-Cvitanovic A, Horzic D, Komes D: Comparative study of polyphenols and caffeine in different coffee varieties affected by the degree of roasting. Food Chemistry 2011, 129:991-1000.

87. Barone JJ, Roberts HR: Caffeine consumption. Food and Chemical Toxicology 1996, 34(1):119-129. 
88. Chin JM, Merves ML, Goldberg BA, Sampson-Cone A, Cone EJ: Caffeine content of brewed teas. Journal of Analytical Toxicology $2008,32: 702-704$.

\section{Figures}

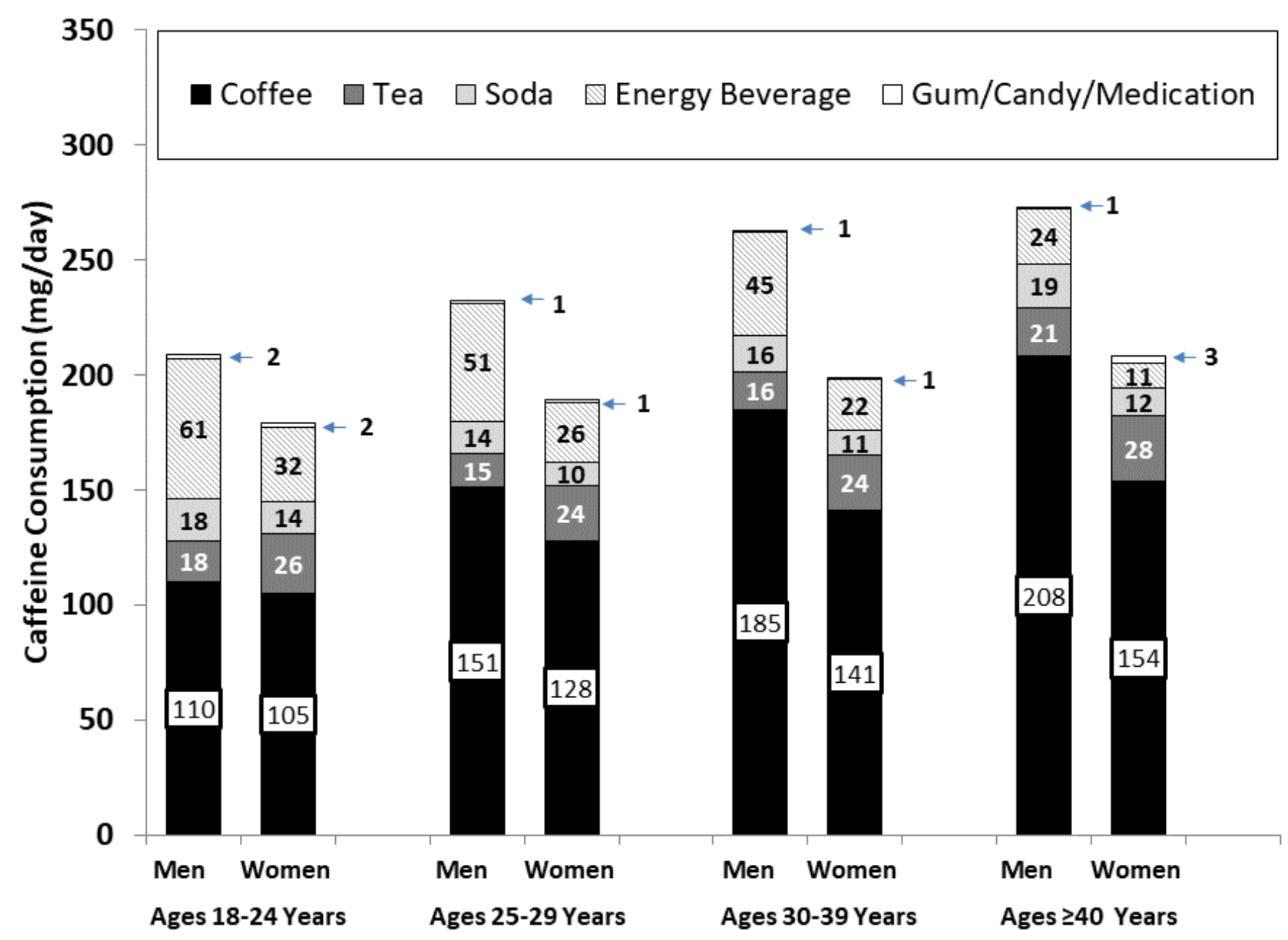

Figure 1

Daily Average Consumption of Caffeinated Substances among Military Personnel who Consume Caffeine by Gender and Age

\section{Supplementary Files}

This is a list of supplementary files associated with this preprint. Click to download.

- DSAECaffeineAllServicesISTROBEStatement.docx 